COMMUNICATIONS IN

ANALYSIS AND GEOMETRY

Volume 12, Number 1, 345-388, 2004

\title{
$1+1$ Wave Maps into Symmetric Spaces
}

\author{
Chuu-Lian Terng ${ }^{1}$ And Karen Uhlenbeck ${ }^{2}$
}

We explain how to apply techniques from integrable systems to construct $2 k$-soliton homoclinic wave maps from the periodic Minkowski space $S^{1} \times R^{1}$ to a compact Lie group, and more generally to a compact symmetric space. We give a correspondence between solutions of the -1 flow equation associated to a compact Lie group $G$ and wave maps into $G$. We use Bäcklund transformations to construct explicit $2 k$-soliton breather solutions for the -1 flow equation and show that the corresponding wave maps are periodic and homoclinic. The compact symmetric space $G / K$ can be embedded as a totally geodesic submanifold of $G$ via the Cartan embedding. We prescribe the constraint condition for the -1 flow equation associated to $G$ which insures that the corresponding wave map into $G$ actually lies in $G / K$. For example, when $G / K=S U(2) / S O(2)=S^{2}$, the constrained -1-flow equation associated to $S U(2)$ has the sine-Gordon equation (SGE) as a subequation and classical breather solutions of the SGE are 2soliton breathers. Thus our result generalizes the result of Shatah and Strauss that a classical breather solution of the SGE gives rise to a periodic homoclinic wave map to $S^{2}$. When the group $G$ is non-compact, the bi-invariant metric on $G$ is pseudo-Riemannian and Bäcklund transformations of a smooth solution often are singular. We use Bäcklund transformations to show that there exist smooth initial data with constant boundary conditions and finite energy such that the Cauchy problem for wave maps from $R^{1,1}$ to the pseudo-Riemannian manifold $S L(2, R)$ develops singularities in finite time.

\footnotetext{
${ }^{1}$ Research supported in part by NSF Grant DMS-0306446.

${ }^{2}$ Research supported in part by Sid Richardson Regents' Chair Funds, University of Texas system and NSF Grant DMS-0305505.
} 


\section{Introduction.}

A smooth map $s: M \rightarrow N$ between two pseudo-Riemannian manifolds is called harmonic if it is a critical point of the functional

$$
J(s)=\frac{1}{2} \int_{M}\left\langle d s_{p}, d s_{p}\right\rangle_{p} d v
$$

where $\langle,\rangle_{p}$ is the natural bilinear form induced from the metrics on $T M_{p}$ and $T N_{s(p)}$, and $d v$ is the volume form of $M$ associated to its metric. When the domain manifold is Riemannian, the Euler-Lagrange equation of $J$ is elliptic, and is the natural non-linear generalization of the Laplace-Beltrami equation. There is an extensive literature in geometry and physics on elliptic harmonic maps. When the domain manifold is the Lorentz space $M=$ $\mathbb{R}^{n, 1}$, the equations are non-linear wave equations, and the solution maps are referred to as wave maps. When the target manifold $N$ is a Lie Group $G$, these equations have a particularly simple form. For $M=\mathbb{R}^{1,1}$, the equation for $s: \mathbb{R}^{1,1} \rightarrow G$ is

$$
\left(s^{-1} s_{t}\right)_{t}=\left(s^{-1} s_{x}\right)_{x}
$$

and a solution is called a $1+1$ wave map into $G$. This equation in light cone (characteristic) coordinates

$$
\xi=\frac{x+t}{2}, \quad \eta=\frac{x-t}{2},
$$

takes the form

$$
\left(s^{-1} s_{\xi}\right)_{\eta}=-\left(s^{-1} s_{\eta}\right)_{\xi}
$$

which can be encoded in a Lax pair, i.e., $s$ is a $1+1$ wave map if and only if

$$
\left[\frac{\partial}{\partial \xi}+\frac{(1-\lambda)}{2} s^{-1} s_{\xi}, \quad \frac{\partial}{\partial \eta}+\frac{\left(1-\lambda^{-1}\right)}{2} s^{-1} s_{\eta}\right]=0
$$

for all $\lambda \in \mathbb{C} \backslash\{0\}$. Because of this Lax formulation, the $1+1$ wave map equation (1.2) is an integrable system.

In this paper we describe how to apply methods from integrable systems to construct periodic and homoclinic wave maps to Lie groups and more generally to symmetric spaces. We will use a closely related integrable nonlinear wave equation, the -1 flow equation associated to $G$. This is the first order semi-linear wave system for $(a, u, v): \mathbb{R}^{1,1} \rightarrow \mathcal{G} \times \mathcal{G} \times \mathcal{G}$ :

$$
\left\{\begin{array}{l}
a_{t}=a_{x}, \\
u_{t}=u_{x}-[a, v], \\
v_{t}=-v_{x}-[u, v],
\end{array}\right.
$$


where $\mathcal{G}$ is the Lie algebra of $G$. The -1 flow equation also has a Lax pair, namely $(a, u, v)$ is a solution of the -1 flow equation if and only if

$$
\left[\frac{\partial}{\partial \xi}+a \lambda+u, \quad \frac{\partial}{\partial \eta}+\lambda^{-1} v\right]=0
$$

for all $\lambda \in \mathbb{C} \backslash\{0\}$, where $\xi, \eta$ are characteristic coordinates. The name -1 flow comes from the standard convention in the theory of soliton equations and we give a brief explanation next.

There is a hierarchy of soliton flows associated to each Lie group $G$. The Lax pair of the $j$-th flow in the $G$-hierarchy is of the form

$$
\left[\frac{\partial}{\partial x}+a \lambda+u, \frac{\partial}{\partial t}+b \lambda^{j}+Q_{1} \lambda^{j-1}+\cdots+Q_{j}\right]=0
$$

with $a, b, u, Q_{i}$ in $\mathcal{G}$. For example, the focusing non-linear Schrödinger equation (NLS)

$$
q_{t}=\frac{i}{2}\left(q_{x x}+2|q|^{2} q\right)
$$

and the complex modified $\mathrm{KdV}$ equation

$$
q_{t}=-\frac{1}{4}\left(q_{x x x}+6|q|^{2} q_{x}\right)
$$

are the second and third flows in the $S U(2)$-hierarchy.

The Lax pairs of the wave map equation and the -1 flow equation are gauge equivalent, which in turn gives an equivalence between solutions of the -1 flow equation associated to $G$ and wave maps $s: \mathbb{R}^{1,1} \rightarrow G$ with $s(0,0)=$ I the identity. This is analogous to the Hasimoto transformation between the focusing NLS and the Heisenberg magnetic model equation. If a solution of the -1 flow equation associated to $G$ satisfies a certain constraint (a reality condition) coming from an involution $\sigma$ of $G$, then the corresponding wave map to $G$ is in fact a wave map to the symmetric space $G / K$ (here $K$ is the fixed point set of $\sigma)$. When $G=S U(2)$ and $\sigma(g)=\left(g^{t}\right)^{-1}$, the constrained -1-flow equation is equivalent to the equation for wave maps from $\mathbb{R}^{1.1}$ to $S^{2}$. Moreover, this constrained -1 flow equation associated to $S U(2)$ contains the sine-Gordon equation (SGE),

$$
q_{t t}-q_{x x}=\sin q
$$

as a subequation.

Shatah and Strauss prove in [3] that wave maps into $S^{2}$ corresponding to classical breather solutions of the SGE are homoclinic, when viewed as 
wave maps from $S^{1} \times \mathbb{R}^{1}$ to $S^{2}$, in the sense that their limits as $t \rightarrow \infty$ and as $t \rightarrow-\infty$ are the same. Our study of periodic $1+1$ wave maps into symmetric spaces was inspired by their paper.

To explain the method we use to construct homoclinic wave maps from $S^{1} \times \mathbb{R}$ to a symmetric space, we need to give a brief review of Bäcklund transformations in integrable systems (cf. [5]). First note that given smooth $\operatorname{gl}(n, \mathbb{C})$ valued maps $A, B$ on $\mathbb{R}^{2}$, the condition that $A, B$ satisfy

$$
\left[\frac{\partial}{\partial \xi}+A, \frac{\partial}{\partial \eta}+B\right]=0
$$

is equivalent to the existence of the trivialization $E$ such that

$$
E_{\xi}=E A, \quad E_{\eta}=E B, \quad E(0,0)=\mathrm{I} .
$$

Now let $(a, u, v)$ be a solution of the -1 flow, and $E(\xi, \eta, \lambda)$ the trivialization of the corresponding Lax pair, i.e.,

$$
E_{\xi}=E(a \lambda+u), \quad E_{\eta}=\lambda^{-1} E v, \quad E(0,0, \lambda)=\mathrm{I} .
$$

Since the coefficients of the above differential equation are holomorphic in the parameter $\lambda \in \mathbb{C} \backslash\{0\}, E(\xi, \eta, \lambda)$ is holomorphic in $\lambda \in \mathbb{C} \backslash\{0\}$. The basic idea of a Bäcklund transformation is that given a linear fractional map from $S^{2}=\mathbb{C} \cup\{\infty\}$ to $G L(n, \mathbb{C})$ of the form $g(\lambda)=\mathrm{I}+\frac{P}{\lambda-z}$ for some $z \in \mathbb{C}$ and $P \in g l(n)$, we can use residue calculus to choose a $g l(n)$-valued map $\tilde{P}$ defined in an open neighborhood $\mathcal{O}$ of $(0,0)$ in the $(\xi, \eta)$-plane so that

$$
\tilde{E}(\xi, \eta, \lambda)=g(\lambda) E(\xi, \eta, \lambda) \tilde{g}(\xi, \eta, \lambda)^{-1}
$$

is holomorphic in $\lambda \in \mathbb{C} \backslash\{0\}$ for each $(\xi, \eta) \in \mathcal{O}$, where $\tilde{g}=I+\frac{\tilde{P}}{\lambda-z}$. By a direct computation, one can see that

$$
\tilde{E}^{-1} \tilde{E}_{\xi}=a \lambda+\tilde{u}, \quad \tilde{E}^{-1} \tilde{E}_{\eta}=\lambda^{-1} \tilde{v}
$$

for some $\tilde{u}, \tilde{v}$ defined on $\mathcal{O}$. Hence $(a, \tilde{u}, \tilde{v})$ is again a solution of the -1 equation. We call

$$
(a, u, v) \mapsto g \bullet(a, u, v):=(a, \tilde{u}, \tilde{v})
$$

a Bäcklund transformation of the -1 flow equation. We notice that the classical breather solutions of SGE can be constructed from the vacuum solution $q=0$ by applying Bäcklund transformations twice with carefully placed poles. Therefore we can apply Bäcklund transformations $2 k$ times to 
construct $2 k$-soliton breathers for SGE. We show that corresponding wave maps into $S^{2}$ are also homoclinic. In fact, we generalize results of Shatah and Strauss to $1+1$ wave maps into any compact symmetric spaces.

Note that if $a \in \mathcal{G}$ is a constant, then $(a, 0, a)$ is a trivial solution of the -1 flow equation, and the $k$-soliton solutions of the -1 flow equation can be constructed by applying Bäcklund transformations to it $k$ times. If we choose $a$ so that $\exp (2 \pi a)=\mathrm{I}$ and place the poles of the Bäcklund transformations carefully, then we can obtain $k$-solitons of the -1 -flow equations, that are periodic in time or in space. Such solutions are called $k$-soliton breathers. The wave maps into $G$ corresponding to $k$-soliton breathers are periodic in the space variable. Wave maps from $S^{1} \times \mathbb{R}$ to $G$ are called periodic wave maps into $G$. The wave map corresponding to the trivial solution $(a, 0, a)$ of the -1 flow equation is a stationary wave map into $G$, which is the geodesic $\gamma(x)=\exp (a x)$ in $G$. We apply Bäcklund transformations to these solutions to construct explicit $k$-soliton periodic wave maps. Moreover, we compute the asymptotic behavior of these periodic wave maps and prove that they are homoclinic. We also construct explicitly $2 k$-soliton homoclinic wave maps from $S^{1} \times \mathbb{R}$ into $\mathbb{C} P^{n}$ and $4 k$-soliton homoclinic wave maps into $S^{n-1}$.

When $G$ is compact, a wave map from $\mathbb{R}^{1,1}$ to $G$ corresponding to a general $k$-soliton solution of the -1 flow equation usually oscillates as the space variable $|x| \rightarrow \infty$, i.e., does not have constant boundary conditions at infinity. To construct wave maps into $G$ that have good boundary conditions and finite energy, we first note that a wave map to a circle subgroup $T$ of $G$ is given essentially by a solution of the linear wave equation, so wave maps to $T$ with finite energy and good boundary conditions at $\pm \infty$ can be written down easily, and Bäcklund transformations of such wave maps are again wave maps having finite energy and constant boundary conditions at infinity.

The Lax pair of the defocusing NLS

$$
q_{t}=\frac{i}{2}\left(q_{x x}-2|q|^{2} q\right)
$$

satisfies the reality condition coming from the non-compac group $S U(1,1)$, and does not have smooth solitons. The theorem that Bäcklund transformations do not introduce singularities applies only to the reality condition coming from a compact Lie group. Unfortunately there are many interesting geometric problems in integrable systems for which solutions obtained via Bäcklund transformations do have singularities. Nevertheless, Bäcklund transformations can still be used in the non-compact case to construct interesting examples as we will see next with $G=S L(2, \mathbb{R})$. 
It is known that the Cauchy problem for wave maps from $\mathbb{R}^{1,1}$ to a complete Riemannian manifold $N$ with smooth initial data in $L_{1}^{2}$ has long time existence (cf. [1]). But this is no longer true if we replace $N$ by a pseudo-Riemannian manifold. There are counterexamples for $N=S L(2, \mathbb{R})$ equipped with the pseudo-Riemannian bi-invariant metric.

This paper is organized as follows. In section 2, we review the Lagrangian formulation of wave maps from $\mathbb{R}^{1,1}$ to $G$ and the corresponding Lax pair. In section 3, we give the Hamiltonian formalism for wave maps and compute the stable and unstable modes at stationary solutions. In section 4 , we prove the Lax pair of the -1 flow equation associated to $G$ is gauge equivalent to the Lax pair of the equation for wave maps into $G$, and give a bijection between solutions of the -1 flow equation and wave maps $s$ satisfying $s(0,0)=\mathrm{I}$. In section 5, we review Bäcklund transformations for the -1 flow equation associated to $S U(n)$, and apply these transformations to stationary wave maps to construct explicit $k$-soliton wave maps from $S^{1} \times \mathbb{R}$ to $S U(2)$. In section 6 , we prove the wave maps to $S U(2)$ corresponding to $2 k$-soliton breather solutions are homoclinic. In section 7 , we explain the constraint condition for the -1 flow equation associated to $S U(2)$ so that the corresponding wave maps into $S U(2)=S^{3}$ lie in $S^{2}$. In section 8, we first recall a useful description of the compact symmetric space $G / K$ imbedded as a totally geodesic submanifold in $G$, and then prescribe the constraint condition for the -1 flow associated to $G$ that insures that the corresponding wave maps into $G$ actually lie in a symmetric space. We emphasize the important case of wave maps into $S^{2}=\frac{S U(2)}{S O(2)}$, into $\mathbb{C} P^{n-1}$, and into $S^{n-1}$. Finally in section 9 , we use Bäcklund transformations to construct examples of smooth Cauchy data with constant boundary conditions at infinity and finite energy such that the Cauchy problem for wave maps from $\mathbb{R}^{1,1}$ to $S L(2, \mathbb{R})$ have long time existence and also examples of initial data that develop singularities in finite time.

\section{Wave map equation and its Lax pair.}

We rewrite (1.3) as a first order system. Let $P=s^{-1} s_{\xi}$, and $Q=s^{-1} s_{\eta}$, i.e.,

$$
s_{\xi}=s P, \quad s_{\eta}=s Q .
$$

The compatibility condition of the linear system (2.1) is

$$
Q P+P_{\eta}=P Q+Q_{\xi},
$$

or equivalently,

$$
P_{\eta}-Q_{\xi}=P Q-Q P=[P . Q] .
$$


Combine equations (2.2) and (1.3) to see that the wave map equation in characteristic coordinates is

$$
P_{\eta}=-Q_{\xi}=\frac{1}{2}[P, Q],
$$

i.e.,

$$
\left(s^{-1} s_{\xi}\right)_{\eta}=-\left(s^{-1} s_{\eta}\right)_{\xi}=\frac{1}{2}\left[s^{-1} s_{\xi}, s^{-1} s_{\eta}\right] .
$$

In other words, we have

Proposition 2.1. Let $(\xi, \eta)$ denote the light cone coordinate system of $\mathbb{R}^{1,1}$. If $s: \mathbb{R}^{1.1} \rightarrow S U(n)$ is a wave map, then $A=\frac{1}{2} s^{-1} s_{\xi}$ and $B=\frac{1}{2} s^{-1} s_{\eta}$ satisfy the first order system

$$
A_{\eta}=-B_{\xi}=[A, B]
$$

Conversely, if $(A, B)$ is a solution of (2.4), then there exists a unique $s$ : $\mathbb{R}^{1,1} \rightarrow S U(n)$ such that

$$
s_{\xi}=2 s A, \quad s_{\eta}=2 s B, \quad s(0,0)=I .
$$

Moreover, s satisfies (1.3), i.e., $s$ is a wave map.

Next we formulate equation (2.4) as the condition for a family of connections to be flat. Recall that the curvature of a $g l(n)$-valued connection

$$
\left\{\frac{\partial}{\partial \xi}+P, \quad \frac{\partial}{\partial \eta}+Q\right\}
$$

is defined to be

$$
F=\left[\frac{\partial}{\partial \xi}+P, \frac{\partial}{\partial \eta}+Q\right]=-P_{\eta}+Q_{\xi}+[P, Q] .
$$

The connection is flat if the curvature is zero. So the compatibility condition (2.2) for linear system (2.1) is also the condition for the connection $\left\{\frac{\partial}{\partial \xi}+P, \frac{\partial}{\partial \eta}+Q\right\}$ to be flat. Another convenient way to write connection is as a $g l(n)$-valued 1 -form

$$
\theta=A d \xi+B d \eta
$$

Then the curvature is

$$
d \theta+\theta \wedge \theta=\left(-A_{\eta}+B_{\xi}+[A, B]\right) d \xi \wedge d \eta .
$$

It is easy to see that the following statements are equivalent for smooth maps $A, B: \mathbb{R}^{2} \rightarrow g l(n)$ : 
1. $\left[\frac{\partial}{\partial x}+A(x, t), \frac{\partial}{\partial t}+B(x, t)\right]=0$,

2. The connection 1-form $\theta=A d x+B d t$ is flat, i.e., $d \theta=-\theta \wedge \theta$.

3. $A_{t}-B_{x}=[A, B]$.

4. $E_{x}=E A, \quad E_{t}=E B, \quad E(0,0)=I$ has a unique solution $E: \mathbb{R}^{2} \rightarrow$ $G L(n)$. Such $E$ is called the trivialization of the flat connection $A d x+$ $B d t$ (normalized at $(0,0)$ ).

The wave map equation has a Lax pair (cf. [7, 6]), i.e., there is a one parameter family of $\operatorname{sl}(n, \mathbb{C})$-valued connection 1 -forms $\Omega_{\lambda}$ on $\mathbb{R}^{1,1}$ defined in terms of $s: \mathbb{R}^{1,1} \rightarrow S U(n)$ and its derivatives so that $\Omega_{\lambda}$ is flat for all $\lambda \in \mathbb{C} \backslash\{0\}$ if and only if $s$ satisfies (1.3). We explain this next. Given $A, B: \mathbb{R}^{1,1} \rightarrow s u(n)$ and $\lambda \in \mathbb{C} \backslash\{0\}$, consider the following $g l(n, \mathbb{C})$-valued connection 1 -form on $\mathbb{R}^{1,1}$ :

$$
\Omega_{\lambda}=(1-\lambda) A d \xi+\left(1-\lambda^{-1}\right) B d \eta .
$$

We claim that $\Omega_{\lambda}$ is flat for all $\lambda \in \mathbb{C}^{*}$ if and only if $(A, B)$ is a solution of (2.4). To see this, note that $\Omega_{\lambda}$ is flat is equivalent to

$$
(1-\lambda) A_{\eta}-\left(1-\lambda^{-1}\right) B_{\xi}=\left[(1-\lambda) A,\left(1-\lambda^{-1}\right) B\right]=\left(2-\lambda-\lambda^{-1}\right)[A, B]
$$

for all $\lambda \in \mathbb{C} \backslash\{0\}$. Equate the coefficients of $\lambda^{-1}, \lambda$ and constant term to get

$$
\left\{\begin{array}{l}
A_{\eta}=[A, B], \\
A_{\eta}-B_{\xi}=2[A, B], \\
B_{\xi}=-[A, B] .
\end{array}\right.
$$

This is equivalent to (2.4), and we prove the claim. We summarize our discussions:

Proposition 2.2. ([6, 7]). Let $s: \mathbb{R}^{1,1} \rightarrow S U(n)$ be a smooth map, $(\xi, \eta)$ the light cone coordinate system, and

$$
A=\frac{1}{2}\left(s^{-1} s_{\xi}\right), \quad B=\frac{1}{2}\left(s^{-1} s_{\eta}\right) .
$$

Then the following statements are equivalent:

1. $s$ is a wave map.

2. $s$ is a solution of (1.3). 
3. $(A, B)$ is a solution of $(2.4)$.

4. The connection 1-form

$$
\Omega_{\lambda}=(1-\lambda) A d \xi+\left(1-\lambda^{-1}\right) B d \eta
$$

is flat for all $\lambda \in \mathbb{C} \backslash\{0\}$,

Corollary 2.3. If $A, B: \mathbb{R}^{1,1} \rightarrow$ su(n) satisfy equation (2.4), then there exists $E(x, t, \lambda)$ such that

$$
E^{-1} E_{\xi}=(1-\lambda) A, \quad E^{-1} E_{\eta}=\left(1-\lambda^{-1}\right) B, \quad E(0,0, \lambda)=I
$$

for all $\lambda \in \mathbb{C} \backslash\{0\}$, i.e., $E(\cdot, \cdot, \lambda)$ is the trivialization of the Lax pair $\Omega_{\lambda}$ defined by (2.5). Moreover, $s(\xi, \eta)=E(\xi, \eta,-1)$ is a wave map from $\mathbb{R}^{1,1}$ to $S U(n), s^{-1} s_{\xi}=2 A$, and $s^{-1} s_{\eta}=2 B$.

A direct computation implies that the Lax pair $\Omega_{\lambda}$ of the wave map equation satisfies the following reality condition:

$$
\Omega_{\bar{\lambda}}^{*}+\Omega_{\lambda}=0,
$$

where $\xi^{*}=\bar{\xi}^{t}$. We claim that the trivialization $E(x, t, \lambda)$ of $\Omega_{\lambda}$ satisfies the reality condition

$$
E(x, t, \bar{\lambda})^{*} E(x, t, \lambda)=I,
$$

or equivalently,

$$
E(x, t, \lambda)^{-1}=E(x, t, \bar{\lambda})^{*} .
$$

To see this, let $F(x, t, \lambda)=\left(E(x, t, \bar{\lambda})^{*}\right)^{-1}$. A direct computation implies that $F^{-1} d F=-\Omega_{\bar{\lambda}}^{*}$, which is equal to $\Omega_{\lambda}$. But $F(0,0, \lambda)=\mathrm{I}$. Since both $E$ and $F$ satisfy the same linear differential equation with the same initial condition, the uniqueness of ODE implies that $E=F$. This proves the claim.

The $\lambda$ parameter seems redundant. But it is this parameter that allows us to construct Bäcklund transformations and explicit solutions. These will be explained in later sections.

\section{The Hamiltonian formulation of wave maps.}

The functional $J$ defined by (1.1) for maps $s: S^{1} \times \mathbb{R} \rightarrow S U(n)$ is

$$
J(s)=\frac{1}{2} \int_{\mathbb{R}^{2}}\left\|s^{-1} s_{t}\right\|^{2}-\left\|s^{-1} s_{x}\right\|^{2} d x d t,
$$


where $\|y\|^{2}=-\operatorname{tr}\left(y^{2}\right)$. Viewed as a functional on the space of curves from $\mathbb{R}$ to $C^{\infty}\left(S^{1}, S U(n)\right), J$ has two terms. The first term of $J$ is the kinetic energy and the second term is the potential energy. The Lagrangian formulation of the wave map equation views the equation as an equation for curves on the tangent bundle of $\mathcal{M}=C^{\infty}\left(S^{1}, S U(n)\right)$. In this section, we use the Legendre transformation to view the wave map equation as a Hamiltonian system on the cotangent bundle of $\mathcal{M}$, and compute the stable and unstable modes at stationary wave maps.

Recall that the cotangent bundle $T^{*} \mathcal{M}$ of a manifold $\mathcal{M}$ has a natural symplectic form $w=d \tau$, where $\tau$ is the canonical 1-form on $T^{*} \mathcal{M}$ defined by

$$
\tau_{\ell}(v)=\ell(d \pi(v))
$$

where $\pi: T^{*} \mathcal{M} \rightarrow \mathcal{M}$ is the natural projection.

Given a curve $\gamma:(-\epsilon, \epsilon) \rightarrow C^{\infty}\left(S^{1}, S U(n)\right)$ with $\gamma(0)=s$, we identify the tangent vector $\gamma^{\prime}(0)$ as

$$
\left(\gamma(0), \gamma(0)^{-1} \gamma^{\prime}(0)\right)=\left(s, s^{-1} \delta s\right)
$$

This identifies $T \mathcal{M}=\mathcal{M} \times C^{\infty}\left(S^{1}, s u(n)\right)$. Note that

$$
\left(v_{1}, v_{2}\right)=-\operatorname{tr}\left(v_{1} v_{2}\right)
$$

defines an inner product on $s u(n)$. So we can also identify $T^{*} \mathcal{M}_{s}$ as $T \mathcal{M}_{s}$ via the $L^{2}$ inner product:

$$
\left\langle v_{1}, v_{2}\right\rangle=\int_{0}^{2 \pi}-\operatorname{tr}\left(v_{1} v_{2}\right) d x
$$

By definition of the canonical 1-form on $T^{*} \mathcal{M}$, we get

$$
\tau_{(s, v)}\left(s^{-1} \delta s, \delta v\right)=\left\langle v, s^{-1} \delta s\right\rangle=\int_{0}^{2 \pi}-\operatorname{tr}\left(v s^{-1} \delta s\right) d x .
$$

We use the Cartan formula

$$
w(X, Y)=d \tau(X, Y)=X(\tau(Y))-Y(\tau(X))-\tau([X, Y])
$$

to compute the symplectic form $w=d \tau$ on $T^{*} \mathcal{M}$. Let $X(s, v)=\left(\eta_{1}, \delta_{1} v\right)$ and $Y(s, v)=\left(\eta_{2}, \delta_{2} v\right)$ be two constant vector fields on $T^{*} \mathcal{M}=T \mathcal{M}$. Then

$$
X(\tau(Y))=X\left(\int_{0}^{2 \pi}-\operatorname{tr}\left(v(x) \eta_{2}(x)\right) d x\right)=-\int_{0}^{2 \pi} \operatorname{tr}\left(\delta_{1} v, \eta_{2}\right) d x=\left\langle\delta_{1} v, \eta_{2}\right\rangle
$$


So we get

$$
w_{(s, v)}\left(\left(\eta_{1}, \delta_{1} v\right),\left(\eta_{2}, \delta_{2} v\right)\right)=\left\langle\delta_{1} v, \eta_{2}\right\rangle-\left\langle\delta_{2} v, \eta_{1}\right\rangle .
$$

Consider the Hamiltonian $H: T^{*} \mathcal{M} \rightarrow \mathbb{R}$, which is the sum of kinetic energy and potential energy, i.e.,

$$
H(s, v)=\frac{1}{2}\left(\langle v, v\rangle+\left\langle s^{-1} s_{x}, s^{-1} s_{x}\right\rangle\right)=-\frac{1}{2} \int_{0}^{2 \pi} \operatorname{tr}\left(v^{2}+\left(s^{-1} s_{x}\right)^{2}\right) d x .
$$

The Hamiltonian vector field $X_{H}$ of $H$ is the vector field satisfying

$$
d H_{(s, v)}\left(s^{-1} \delta s, \delta v\right)=w\left(\left(s^{-1} \delta s, \delta v, X_{H}(s, v)\right)\right.
$$

for all $\left(s^{-1} \delta s, \delta v\right)$. A direct computation shows that

$$
d H_{(s, v)}\left(s^{-1} \delta s, \delta v\right)=\langle\delta v, v\rangle-\left\langle s^{-1} \delta s,\left(s^{-1} s_{x}\right)_{x}\right\rangle .
$$

So the Hamiltonian vector field for $H$ is

$$
X_{H}(s, v)=\left(v,\left(s^{-1} s_{x}\right)_{x}\right)
$$

The Hamiltonian equation is

$$
s^{-1} s_{t}=v, \quad v_{t}=\left(s^{-1} s_{x}\right)_{x},
$$

which is the wave map equation (1.2).

Proposition 3.1. The stationary points of $X_{H}$ are $(s, 0)$, where $s(x)=$ $c e^{a x}, a \in s u(2)$ a constant such that $e^{2 \pi a}=I$ and $c \in S U(2)$ a constant.

Proof. $X_{H}(s, v)=(0,0)$ if and only if $v=0$ and $\left(s^{-1} s_{x}\right)_{x}=0$. So $s^{-1} s_{x}=a$ for some constant $a \in s u(n)$. Hence $s(x)=c e^{a x}$ for some $c \in s u(n)$. Since $s(2 \pi)=s(0), e^{2 \pi a}=I$.

Note that stationary points of $X_{H}$ are closed geodesics of $S U(n)$.

Next we compute the linearization of $X_{H}$ at a stationary point, stable and unstable subspaces. We will do this calculation for $S U(2)$. The calculations for other compact groups are similar.

Let $m$ be a non-zero integer, $a=\operatorname{diag}(i m,-i m)$, and $s(x)=e^{a x}$. The linearization of $X=X_{H}$ at the stationary point $(s, 0)$ is

$$
d X_{(s, 0)}\left(s^{-1} \delta s, \delta v\right)=\left(\delta v, \delta\left(s^{-1} s_{x}\right)_{x}\right)
$$


Set

$$
s^{-1} \delta s=p, \quad \delta v=q .
$$

Compute directly to get

$$
\begin{aligned}
\delta\left(s^{-1} s_{x}\right) & =-\left(s^{-1} \delta s\right) s^{-1} s_{x}+s^{-1}(\delta s)_{x} \\
& =-p a+s^{-1}(s p)_{x}=-p a+s^{-1}\left(s_{x} p+s p_{x}\right) \\
& =-p a+a p+p_{x}=p_{x}+[a, p] .
\end{aligned}
$$

So

$$
d X_{(s, 0)}(p, q)=\left(q, p_{x x}+\left[a, p_{x}\right]\right) .
$$

The linearized equation is

$$
\left\{\begin{array}{l}
p_{t}=q \\
q_{t}=p_{x x}+\left[a, p_{x}\right] .
\end{array}\right.
$$

The linearization of the wave map equation at $s$ is

$$
p_{t t}=p_{x x}+\left[a, p_{x}\right]
$$

We compute the linear modes of the linear wave equation (3.1) next, i.e., solve the following linear system for $(p, q): S^{1} \rightarrow s u(2)$ :

$$
\left\{\begin{array}{l}
q=k p, \\
p_{x x}+\left[a, p_{x}\right]=k q .
\end{array}\right.
$$

Substitute the first equation to the second to get

$$
p_{x x}+\left[a, p_{x}\right]-k^{2} p=0 .
$$

Write (3.3) in terms of entries of $p=\left(p_{i j}\right) \in s u(2)$ to get

$$
\left\{\begin{array}{l}
\left(p_{11}\right)_{x x}-k^{2} p_{11}=0, \\
\left(p_{12}\right)_{x x}+2 i m\left(p_{12}\right)_{x}-k^{2} p_{12}=0 .
\end{array}\right.
$$

This system is linear with constant coefficients. So it can be solved explicitly:

$$
\begin{aligned}
p_{11}(x) & = \begin{cases}c_{1}+c_{2} x, & \text { if } k=0 \\
c_{1} e^{k x}+c_{2} e^{-k x}, & \text { if } k \neq 0\end{cases} \\
p_{12} & =c_{1} e^{\left(-i m+\sqrt{k^{2}-m^{2}}\right) x}+c_{2} e^{\left(-i m-\sqrt{k^{2}-m^{2}}\right) x}
\end{aligned}
$$


We divide the computation into three cases:

(1) $k=0$.

Since in this case $p_{11}$ is linear and periodic with period $2 \pi, p_{11}=c_{1}$ is a pure imaginary constant. Note $p_{12}=c_{2}+c_{3} e^{-2 i m x}$ with $c_{2}, c_{3} \in \mathbb{C}$. So the nullity of $d\left(X_{H}\right)_{(s, 0)}$ is 5 . In fact, let $s_{c b}$ denote the stationary point $s_{c, b}(x)=c e^{b x}$. Then $\left\{s_{c, b} \mid c \in S U(2), b \in s u(2)\right.$ is conjugate to $\left.a\right\}$ is a five dimensional stationary submanifold of $\mathcal{M}$ and the tangent space at $s=s_{\mathrm{I}, a}$ is the kernel of the linearization (3.2).

(2) $k \in \mathbb{R} \backslash\{0\}$.

Note $p_{11}=c_{1} e^{k x}+c_{2} e^{-k x}$ is periodic and $k$ is non-zero and real implies that $p_{11}=0$. Since $p_{12}$ has period $2 \pi, m^{2}-k^{2} \geq 0$ and $\sqrt{m^{2}-k^{2}}$ is an integer. So real non-zero eigenvalues of $d\left(X_{H}\right)_{(s, 0)}$ are

$$
k= \pm \sqrt{m^{2}-j^{2}}, \quad 0 \leq|j|<m, \quad j \text { integer }
$$

Eigenvectors for $k= \pm \sqrt{m^{2}-j^{2}}$ are $\left(p_{k}, q_{k}^{ \pm}\right)$, where

$$
\begin{aligned}
p_{k} & =\left(\begin{array}{cc}
0 & c_{1} e^{-i(m+j) x}+c_{2} e^{-i(m-j) x} \\
-\bar{c}_{1} e^{i(m+j) x}-\bar{c}_{2} e^{i(m-j) x} & 0
\end{array}\right), \\
q_{k}^{ \pm} & = \pm \sqrt{m^{2}-j^{2}} p_{k} .
\end{aligned}
$$

(3) $k \in \mathbb{C} \backslash \mathbb{R}$.

Since $p_{12}$ has to be periodic, $m^{2}-k^{2}>0$ and $\sqrt{m^{2}-k^{2}}$ is an integer. Hence $k^{2}$ must be real. But $k$ is not real. So $k$ is pure imaginary. In other words, $k=i c$ for some $c \in \mathbb{R}$ and $\sqrt{m^{2}+c^{2}}$ is an integer. Hence the non-real eigenvalues are

$$
\pm i \sqrt{j^{2}-m^{2}}, \quad j>|m|, \quad j \text { integer. }
$$

Recall that the stable (unstable resp.) subspace of $X_{H}$ at a stationary point $(s, 0)$ is the direct sum of the eigenspaces of $d\left(X_{H}\right)_{(s, 0)}$ with eigenvalues $k$ such that $\operatorname{Re}(k)<0(\operatorname{Re}(k)>0$ resp.) So the above computation gives

Proposition 3.2. Let $m$ be an integer, $a=\operatorname{diag}(i m,-i m)$, and $s(x)=e^{a x}$. The unstable subspace of the Hamiltonian vector field $X_{H}$ at the stationary point $(s, 0)$ is $\oplus_{j=0}^{m-1} W_{j}^{+}$, where $W_{j}^{+}$is the eigenspace of $d\left(X_{H}\right)_{(s, 0)}$ with eigenvalue $k=\sqrt{m^{2}-j^{2}}$ and is spanned by $\left(p_{k}, q_{k}^{+}\right)$given in (3.5). The stable subspace of the Hamiltonian vector field $X_{H}$ at $(s, 0)$ is $\oplus_{j=0}^{m-1} W_{j}^{-}$, where $W_{j}^{-}$is the eigenspace of $d\left(X_{H}\right)_{(s, 0)}$ with eigenvalue $k=-\sqrt{m^{2}-j^{2}}$ and is spanned by $\left(p_{k}, q_{k}^{-}\right)$given in (3.5). 
Corollary 3.3. Let $m$ be a positive integer, and $a=\operatorname{diag}(i m,-i m)$. Then the linearization of the wave map equation at the stationary wave map $s(x, t)$ $=e^{a x}$ is

$$
\xi_{t t}=\xi_{x x}+\left[a, \xi_{x}\right] .
$$

Moreover, the stable and unstable modes corresponding to $\pm \sqrt{m^{2}-j^{2}}$ are

$$
\begin{gathered}
p_{m, j}^{-}(x, t)=-e^{-\sqrt{m^{2}-j^{2}} t}\left(\begin{array}{cc}
0 & c e^{i(-m \pm j) x} \\
-\bar{c} e^{-i(-m \pm j) x} & 0
\end{array}\right) \\
p_{m, j}^{+}(x, t)=e^{\sqrt{m^{2}-j^{2}} t}\left(\begin{array}{cc}
0 & c e^{i(-m \pm j) x} \\
-\bar{c} e^{-i(-m \pm j) x} & 0
\end{array}\right)
\end{gathered}
$$

respectively, where $c \in \mathbb{C}$ is a constant, $j$ is an integer and $|j|<m$.

\section{The -1 flow equation and the wave map.}

We give a correspondence between solutions of the -1 flow equation (1.4) and wave maps.

In characteristic coordinate $(\xi, \eta)$, the -1 flow equation (1.4) associated to $S U(n)$ is the following system for $(a, u, v): \mathbb{R}^{2} \rightarrow \Pi_{i=1}^{3} s u(n)$ :

$$
\left\{\begin{array}{l}
a_{\eta}=0, \\
u_{\eta}=[a, v], \\
v_{\xi}=-[u, v] .
\end{array}\right.
$$

A direct computation implies that

Proposition 4.1. The map $(a, u, v): \mathbb{R}^{2} \rightarrow \Pi_{i=1}^{3} s u(n)$ is a solution of the -1-flow equation (4.1) associated to $S U(n)$ if and only if

$$
\theta_{\lambda}=(a \lambda+u) d \xi+\lambda^{-1} v d \eta
$$

is flat for all $\lambda \in \mathbb{C} \backslash\{0\}$.

Note that the Lax pair $\theta_{\lambda}$ of the above -1 flow equation satisfies the reality condition (2.6). So the trivialization $E(x, t, \lambda)$ of $\theta_{\lambda}$ satisfies the reality condition $(2.7)$.

Recall that the gauge transformation of $g: \mathbb{R}^{1,1} \rightarrow G L(n, \mathbb{C})$ of the connection

$$
\left\{\frac{\partial}{\partial \xi}+A, \quad \frac{\partial}{\partial \eta}+B\right\}
$$


is

$$
\left\{g\left(\frac{\partial}{\partial \xi}+A\right) g^{-1}, \quad g\left(\frac{\partial}{\partial \eta}+B\right) g^{-1}\right\} .
$$

Direct computation gives

$$
\begin{aligned}
& g\left(\frac{\partial}{\partial \xi}+A\right) g^{-1}=\frac{\partial}{\partial \xi}+g A g^{-1}-g_{\xi} g^{-1} \\
& g\left(\frac{\partial}{\partial \eta}+B\right) g^{-1}=\frac{\partial}{\partial \eta}+g B g^{-1}-g_{\eta} g^{-1} .
\end{aligned}
$$

Since

$$
\left[g\left(\frac{\partial}{\partial \xi}+A\right) g^{-1}, \quad g\left(\frac{\partial}{\partial \eta}+B\right) g^{-1}\right]=g\left[\frac{\partial}{\partial \xi}+A, \frac{\partial}{\partial \eta}+B\right] g^{-1},
$$

the gauge transformation of a flat connection is again flat. Written in terms of connection 1-form $\theta=A d \xi+B d \eta$, the gauge transformation $g * \theta$ is

$$
g * \theta=g \theta g^{-1}-d g g^{-1} .
$$

It is easy to check that if $E$ is the trivialization of the flat connection $\theta$, then $g(0,0)^{-1} E g^{-1}$ is the trivialization of $g * \theta$.

Below we show that the Lax pairs of wave map equation and the -1 flow equation are gauge equivalent and give a correspondence between wave maps and solutions of the -1 flow equation.

Theorem 4.2. Let $(a, u, v)$ be a solution to the -1-flow equation (4.1) associated to $S U(n)$, and $\Phi(\xi, \eta, \lambda)$ the trivialization of

$$
\theta_{\lambda}=(a(\xi, \eta) \lambda+u(\xi, \eta)) d \xi+\lambda^{-1} v(\xi, \eta) d \eta .
$$

Set $\Phi(\lambda)(\xi, \eta)=\Phi(\xi, \eta, \lambda)$. Then $s=\Phi(-1) \Phi(1)^{-1}$ is a wave map from $\mathbb{R}^{1,1}$ to $S U(n)$, and

$$
s^{-1} s_{\xi}=-2 \Phi(1) a \Phi(1)^{-1}, \quad s^{-1} s_{\eta}=-2 \Phi(1) v \Phi(1)^{-1} .
$$

Conversely, suppose $s: \mathbb{R}^{1,1} \rightarrow S U(n)$ is a wave map and $s(0,0)=\mathrm{I}$. Let $\psi(\xi, \eta)$ be the solution of $\psi^{-1} \psi_{\eta}=\frac{1}{2} s^{-1} s_{\eta}$ with $\psi(\xi, 0)=\mathrm{I}$, and

$$
\left\{\begin{array}{l}
a(\xi, \eta)=\frac{1}{2}\left(s^{-1} s_{\xi}\right)(\xi, 0), \\
u(\xi, \eta)=a(\xi)-\left(\psi_{\xi} \psi^{-1}\right)(\xi, \eta), \\
v=-\frac{1}{2} \psi s^{-1} s_{\eta} \psi^{-1}
\end{array}\right.
$$

Then $(-a, u, v)$ is a solution of the -1 flow equation associated to $S U(n)$ and $s$ is the wave map corresponding to $(-a, u, v)$. 
Proof. A direct computation gives

$\Theta_{\lambda}=\Phi(1) * \theta_{\lambda}=(1-\lambda)\left(-\Phi(1) a \Phi(1)^{-1}\right) d \xi+\left(1-\lambda^{-1}\right)\left(-\Phi(1) v \Phi(1)^{-1}\right) d \eta$.

The trivialization of $\Theta_{\lambda}$ is $\Phi(\lambda) \Phi(1)^{-1}$. By Corollary $2.2, \Phi(-1) \Phi(1)^{-1}$ is a wave map.

To prove the converse, set $A=\frac{1}{2} s^{-1} s_{\xi}$ and $B=\frac{1}{2} s^{-1} s_{\eta}$. By Proposition 2.2 , we have $A_{\eta}=-B_{\xi}=[A, B]$. Set $\tilde{A}(\xi, \eta)=\psi(\xi, \eta)^{-1} a(\xi) \psi(\xi, \eta)$. A direct computation implies that $\tilde{A}_{\eta}=[\tilde{A}, B]$. But $A$ satisfies the same differential equation as $\tilde{A}$, i.e., $A_{\eta}=[A, B]$, and

$$
\tilde{A}(\xi, 0)=A(\xi, 0)=a(\xi)=\frac{1}{2}\left(s^{-1} s_{\xi}\right)(\xi, 0) .
$$

By the uniqueness of solutions of ordinary differential equation we have

$$
A(\xi, \eta)=\tilde{A}(\xi, \eta)=\psi^{-1}(\xi, \eta) a(\xi) \psi(\xi, \eta)
$$

Apply gauge transformation of $\psi$ to the Lax pair of the wave map

$$
\Omega_{\lambda}=(1-\lambda) A d \xi+\left(1-\lambda^{-1}\right) B d \eta
$$

to get

$$
\psi * \Omega_{\lambda}=\left(-a \lambda+a-\psi_{\xi} \psi^{-1}\right) d \xi-\lambda^{-1} \psi B \psi^{-1} d \eta .
$$

Since $\Omega_{\lambda}$ is flat. so is $\psi * \Omega_{\lambda}$. It follows from Proposition 4.1 that $(-a, u, v)$ is a solution of the -1 flow, where $a, u, v$ are defined by (4.4).

Let $F(\xi, \eta, \lambda)$ denote the trivialization of $\Omega_{\lambda}$. Since $\Omega_{1}=0, F(\xi, \eta, 1)$ is a constant. But $F(0,0, \lambda)=\mathrm{I}$. Thus $F(\xi, \eta, 1)=\mathrm{I}$. It follows from Corollary 2.3 that the harmonic map $s(\xi, \eta)=F(\xi, \eta,-1)$. The trivialization of $\psi * \Omega_{\lambda}$ is

$$
E(\xi, \eta, \lambda)=F(\xi, \eta, \lambda) \psi(\xi, \eta) .
$$

But we have proved $\psi * \Omega_{\lambda}$ is the Lax pair for $(-a, u, v)$. So the wave map corresponding to $(-a, u, v)$ is

$$
E(\xi, \eta,-1) E(\xi, \eta, 1)^{-1}=F(\xi, \eta,-1) F(\xi, \eta, 1)^{-1}=F(\xi, \eta,-1)=s .
$$

The proof of the above Theorem implies that the Lax pair (2.5) of the wave map equation is gauge equivalent to the Lax pair (4.2) of the -1 flow equation. 


\section{Example 4.3. closed geodesics}

Let $a=\operatorname{diag}\left(i m_{1}, \cdots, i m_{n}\right) \in s u(n)$, where $2 m_{1}, \cdots, 2 m_{n}$ are integers. Then $(a, 0, a)$ is a solution of the -1 flow equation, the corresponding Lax pair is

$$
\theta_{\lambda}=a \lambda d \xi+a \lambda^{-1} d \eta
$$

and the trivialization of $\theta_{\lambda}$ is

$$
E_{0}(\xi, \eta, \lambda)=e^{a\left(\lambda \xi+\lambda^{-1} \eta\right)}
$$

The corresponding wave map constructed in Theorem 4.2 is the stationary wave map

$$
s_{0}(\xi, \eta)=E_{0}(\xi, \eta,-1) E_{0}(\xi, \eta, 1)^{-1}=e^{-2 a x}=\operatorname{diag}\left(e^{-2 i m_{1} x}, \ldots, e^{-2 i m_{n} x}\right),
$$

a closed geodesic in $S U(n)$.

Note that $S U(2)$ equipped with the bi-invariant metric is isometric to the standard $S^{3}$ because

$$
S U(2)=\left\{\left.\left(\begin{array}{cc}
z & -\bar{w} \\
w & \bar{z}
\end{array}\right)|z, w \in \mathbb{C},| z\right|^{2}+|w|^{2}=1\right\}
$$

is isometric to $S^{3}$ in $\mathbb{C}^{2}=\mathbb{R}^{4}$ via

$$
\left(\begin{array}{cc}
z & -\bar{w} \\
w & \bar{z}
\end{array}\right) \mapsto\left(\begin{array}{c}
z \\
w
\end{array}\right)
$$

\section{Example 4.4. wave maps into a great circle}

Let $h(\xi)$ and $k(\eta)$ be smooth real valued functions on $\mathbb{R}, u=0$, and

$$
a=h^{\prime}(\xi)\left(\begin{array}{cc}
i & 0 \\
0 & -i
\end{array}\right), \quad b=k^{\prime}(\eta)\left(\begin{array}{cc}
i & 0 \\
0 & -i
\end{array}\right) .
$$

Then $(a, 0, b)$ is a solution of the -1 flow equation associated to $S U(2)$, its Lax pair is $\theta_{\lambda}=a(\xi) \lambda d \xi+b(\eta) \lambda^{-1} d \eta$, and its trivialization is

$$
E(\xi, \eta, \lambda)=\left(\begin{array}{cc}
e^{i\left(h(\xi) \lambda+k(\eta) \lambda^{-1}\right)} & 0 \\
0 & e^{-i\left(h(\xi) \lambda+k(\eta) \lambda^{-1}\right)}
\end{array}\right)
$$

The wave map corresponding to $(a, 0, b)$ is

$$
s(\xi, \eta)=\operatorname{diag}\left(e^{-2 i(h(\xi)+k(\eta))}, e^{2 i(h(\xi)+k(\eta))}\right),
$$


which lies in the great circle $|z|=1$ and $w=0$ in

$$
S U(2)=\left\{\left.\left(\begin{array}{cc}
z & -\bar{w} \\
w & \bar{z}
\end{array}\right)|z, w \in \mathbb{C},| z\right|^{2}+|w|^{2}=1\right\}=S^{3} .
$$

Hence it is also a wave map into the circle $S^{1}$. The equation for wave maps into $S^{1}$ is essentially the linear wave equation, and general solutions are of the form $h(\xi)+k(\eta)$. Note that if $h$ and $k$ decay at $\pm \infty$, then the corresponding wave map tends to I as $|x| \rightarrow \infty$.

\section{Bäcklund transformations.}

In this section, we use Bäcklund transformations to construct $k$-soliton solutions of the -1 flow equation, and use Theorem 4.2 to construct the corresponding $k$-soliton wave maps. Most of these wave maps oscillates as the space variable $x$ tends to $\pm \infty$, but some of these wave maps are periodic in $x$. Note that wave maps into a great circle of $S U(2)$ can be written in terms of solutions of the linear wave equation. We show that if $s$ is a wave map into a great circle so that $s$ has constant boundary condition at $\pm \infty$ and finite energy, then the new wave maps obtained by applying Bäcklund transformations to $s$ also have constant boundary condition and finite energy.

First we review the construction of Bäcklund transformations of the -1 flow equation. Let $(a, u, v)$ be a solution of the -1 flow equation (4.1) associated to $S U(n)$, and $E(x, t, \lambda)$ the trivialization of its Lax pair (4.2) $\theta_{\lambda}$, i.e.,

$$
\left\{\begin{array}{l}
E^{-1} E_{\xi}=a \lambda+u, \\
E^{-1} E_{\eta}=\lambda^{-1} v, \\
E(0,0, \lambda)=\mathrm{I}
\end{array}\right.
$$

Since the right hand side of (5.1) is holomorphic in parameter $\lambda \in \mathbb{C} \backslash 0$, the solution $E(x, t, \lambda)$ is holomoprhic in $\lambda \in \mathbb{C} \backslash 0$. Because $\theta_{\lambda}$ satisfies the reality condition (2.6), $E$ satisfies (2.7).

Let $\pi$ be a Hermitian projection of $\mathbb{C}^{n}$ onto a complex linear subspace $V$, $\pi^{\perp}=I-\pi$ the projection onto the orthogonal complement $V^{\perp}$, and $z \in \mathbb{C}$. Let $g_{z, \pi}: \mathbb{C} \rightarrow G L(n, \mathbb{C})$ denote the rational map defined by

$$
g_{z, \pi}(\lambda)=\pi+\frac{\lambda-z}{\lambda-\bar{z}} \pi^{\perp}=I+\frac{\bar{z}-z}{\lambda-\bar{z}} \pi^{\perp} .
$$

We call $g_{z, \pi}$ a simple element. A direct computation shows that $g_{z, \pi}$ satisfies 
the reality condition (2.7):

$$
\left(g_{z, \pi}(\bar{\lambda})\right)^{*} g_{z, \pi}(\lambda)=I .
$$

In particular,

$$
g_{z, \pi}^{-1}(\lambda)=\left(g_{z, \pi}(\lambda)\right)^{*}=\pi+\frac{\lambda-\bar{z}}{\lambda-z} \pi^{\perp}=g_{\bar{z}, \pi}(\lambda) .
$$

To construct Bäcklund transformations for the -1 flow equation, we first find $\tilde{E}$ and $\tilde{g}$ so that

$$
g_{z, \pi}(\lambda) E(x, t, \lambda)=\tilde{E}(x, t, \lambda) \tilde{g}(x, t, \lambda)
$$

with $\tilde{E}$ holomorphic in $\lambda \in \mathbb{C} \backslash 0$ and $\tilde{g}$ holomorphic in a neighborhood of $\{0, \infty\}$. Since the left hand side has a pole at $\lambda=\bar{z}$, so $\tilde{g}$ must have too. In fact, $\tilde{g}$ can be taken to be the form $g_{z, \tilde{\pi}(x, t)}(\lambda)$ for some projection $\tilde{\pi}(x, t)$. Moreover, $\tilde{E}$ is the trivialization of a new solution of the -1 flow equation. We state the results more precisely below.

Theorem 5.1. ([5]). Let $(a, u, v)$ be a solution of the -1 flow equation (4.1), and $E(\xi, \eta, \lambda)$ the trivialization of the corresponding Lax pair $\theta_{\lambda}$, i.e.,

$$
E^{-1} E_{\xi}=a \lambda+u, \quad E^{-1} E_{\eta}=\lambda^{-1} v, \quad E(0,0, \lambda)=I .
$$

Let $z \in \mathbb{C} \backslash \mathbb{R}$, and $\pi$ the projection onto a linear subspace $V$ of $\mathbb{C}^{n}$. Set

$$
\begin{aligned}
& \tilde{V}(\xi, \eta)=E(\xi, \eta, z)^{*}(V) \\
& \tilde{\pi}(\xi, \eta)=\text { the Hermitian projection of } \mathbb{C}^{n} \text { onto } \tilde{V}(\xi, \eta) \\
& \tilde{u}=u+(z-\bar{z})[\tilde{\pi}, a] \\
& \tilde{v}=\frac{1}{|z|^{2}}\left(\bar{z} \tilde{\pi}+z \tilde{\pi}^{\perp}\right) v\left(z \tilde{\pi}+\bar{z} \tilde{\pi}^{\perp}\right), \\
& \tilde{E}(\xi, \eta, \lambda)=g_{z, \pi}(\lambda) E(\xi, \eta, \lambda) g_{z, \tilde{\pi}}(\lambda)^{-1} \\
& \quad=\left(\pi+\frac{\lambda-z}{\lambda-\bar{z}} \pi^{\perp}\right) E(\xi, \eta, \lambda)\left(\tilde{\pi}(\xi, \eta)+\frac{\lambda-\bar{z}}{\lambda-z} \tilde{\pi}^{\perp}(\xi, \eta)\right) .
\end{aligned}
$$

Then

1. $\tilde{E}(\xi, \eta, \lambda)$ is holomorphic for $\lambda \in \mathbb{C} \backslash 0$,

2. $(a, \tilde{u}, \tilde{v})$ is a new solution of the -1 flow equation, 
3. $\tilde{E}$ satisfies the reality condition (2.7), and $\tilde{E}$ is the trivialization of the Lax pair of $(a, \tilde{u}, \tilde{v})$.

We sketch the proof of this Theorem. Let $g=g_{z, \pi}$, and $\tilde{g}=g_{z, \tilde{\pi}(x, t)}$. Note that $\tilde{E}=g E \tilde{g}^{-1}$ is holomoprhic for $\lambda \in \mathbb{C} \backslash\{0, z, \bar{z}\}$ and has poles at $\lambda=z$ and $\bar{z}$ of order $\leq 1$. Use definition of $\tilde{\pi}$ to prove that the residues of $\tilde{E}$ at $\lambda=z$ and $\lambda=\bar{z}$ are zero. Thus $\tilde{E}$ is holomorphic for $\lambda \in \mathbb{C} \backslash 0$. Let $\tilde{\theta}_{\lambda}=\tilde{E}^{-1} d \tilde{E}$. Then

$$
\tilde{\theta}_{\lambda}=\tilde{g} \theta_{\lambda} \tilde{g}^{-1}-(d \tilde{g}) \tilde{g}^{-1}=\tilde{g} * \theta_{\lambda}
$$

Expand $\tilde{E}^{-1} \tilde{E}_{\xi}$ in $\lambda$ to see that its leading term is $a \lambda$. Since $\tilde{E}^{-1} \tilde{E}_{\xi}$ is holomorphic in $\lambda \in \mathbb{C}$, it must be of the form $a \lambda+\tilde{u}$ for some $\tilde{u}$. A similar argument implies that $\tilde{E}^{-1} \tilde{E}_{\eta}$ must be of the form $\lambda^{-1} \tilde{v}$. But $\tilde{\theta}_{\lambda}=(a \lambda+$ $\tilde{u}) d \xi+\lambda^{-1} \tilde{v} d \eta$ is flat. So $(a, \tilde{u}, \tilde{v})$ is a solution of the -1 flow equation. The formula of $\tilde{u}, \tilde{v}$ can be computed from (5.3).

Theorem 5.1 gives an algebraic method to construct new solutions from a given solution of the -1 flow equation if the trivialization of the Lax pair of the given solution is known. Let $g_{z, \pi} \bullet(a, u, v)$ denote the solution $(a, \tilde{u}, \tilde{v})$ constructed in Theorem 5.1, and the transformation $(a, u, v) \mapsto g_{z, \pi} \bullet(a, u, v)$ is called a Bäcklund transformation of the -1 flow equation. Let $s$ be the wave map corresponding to $(a, u, v)$ given by Theorem 4.2 , and $g_{z, \pi} \bullet s$ the wave map corresponding to $g_{z, \pi} \bullet(a, u, v)$. We call $s \mapsto g_{z, \pi} \bullet s$ a Bäcklund transformation of wave maps.

In the next two examples, we use Bäcklund transformations to construct explicit wave maps into $S U(2)$.

\section{Example 5.2. periodic 1-soliton wave map}

Let $2 m>0$ be an integer, $a=\operatorname{diag}(i m,-i m)$. We have seen in Example 4.3 that $(a, 0, a)$ is a solution of the -1 flow equation, its Lax pair is

$$
\theta_{\lambda}=a \lambda d \xi+a \lambda^{-1} d \eta
$$

its trivialization is $E_{0}(\xi, \eta, \lambda)=e^{a \lambda \xi+a \lambda^{-1} \eta}$, and the corresponding wave map is the stationary wave map $s_{0}(x, t)=e^{-2 a x}$. Since we have the trivialization for $(a, 0, a)$, we can apply Bäcklund transformation to $(a, 0, a)$. Let $z=e^{i \theta}$, $q_{0}=\left(\begin{array}{l}1 \\ 1\end{array}\right)$, and $\pi$ the Hermitian projection onto $V=\mathbb{C} q_{0}$. We use Theorem 
5.1 to compute $g_{z, \pi} \bullet(a, 0, a)$ next. First we get

$$
\begin{aligned}
\tilde{q}(\xi, \eta) & =\exp \left(a\left(z \xi+z^{-1} \eta\right)\right)^{*} q_{0}=\exp \left(-a\left(\bar{z} \xi+\bar{z}^{-1} \eta\right)\right) q_{0} \\
& =\exp \left(-a\left(e^{-i \theta} \xi+e^{i \theta} \eta\right)\right) q_{0} \\
& =\exp (-a(\cos \theta(\xi+\eta)+i \sin \theta(-\xi+\eta))) q_{0} \\
& =\exp \left(-a(x \cos \theta-i t \sin \theta)\left(\begin{array}{l}
1 \\
1
\end{array}\right)=\left(\begin{array}{c}
e^{-(i m x \cos \theta+m t \sin \theta)} \\
e^{i m x \cos \theta+m t \sin \theta}
\end{array}\right) .\right.
\end{aligned}
$$

Therefore the projection $\tilde{\pi}(x, t)$ of $\mathbb{C}^{2}$ onto $\mathbb{C} \tilde{q}(x, t)$ is

$$
\tilde{\pi}(x, t)=\frac{1}{e^{2 m t \sin \theta}+e^{-2 m t \sin \theta}}\left(\begin{array}{cc}
e^{-2 m t \sin \theta} & e^{-2 i m x \cos \theta} \\
e^{2 i m x \cos \theta} & e^{2 m t \sin \theta}
\end{array}\right),
$$

the trivialization of $g_{z, \pi} \bullet(a, 0, a)$ is

$$
\begin{aligned}
E_{1}(\xi, \eta, \lambda) & =g_{z, \pi}(\lambda) E_{0}(\xi, \eta, \lambda) g_{z \cdot \tilde{\pi}(\xi, \eta)}(\lambda)^{-1} \\
& =\left(\pi+\frac{\lambda-z}{\lambda-\bar{z}} \pi^{\perp}\right) e^{a\left(\lambda \xi+\lambda^{-1} \eta\right)}\left(\tilde{\pi}(x, t)+\frac{\lambda-\bar{z}}{\lambda-z} \tilde{\pi}^{\perp}(x, t)\right) .
\end{aligned}
$$

By Theorem 4.2, the wave map $s$ corresponding to $g_{z, \pi} \bullet(a, 0, a)$ is

$$
\begin{aligned}
& s(x, t)=E_{1}(x, t,-1) E_{1}(x, t, 1)^{-1} \\
& =g_{z, \pi}(-1) E_{0}(x, t,-1) g_{z, \tilde{\pi}(x, t)}(-1)^{-1} g_{z, \tilde{\pi}(x, t)}(1) E_{0}(x, t, 1)^{-1} g_{z, \pi}(1)^{-1} .
\end{aligned}
$$

A direct computation gives

$$
\begin{aligned}
& E_{0}(x, t,-1)=E_{0}(x, t, 1)^{-1}=e^{-a x}, \\
& g_{z, \tilde{\pi}(x, t)}(-1)^{-1} g_{z, \tilde{\pi}(x, t)}(1)=\tilde{\pi}(x, t)-\tilde{\pi}(x, t)^{\perp} .
\end{aligned}
$$

Hence

$$
s(x, t)=g_{z, \pi}(-1) e^{-a x}\left(\tilde{\pi}(x, t)-\tilde{\pi}(x, t)^{\perp}\right) e^{-a x} g_{z, \pi}(1)^{-1} .
$$

In particular, the first column of $e^{-a x}\left(\tilde{\pi}(x, t)-\tilde{\pi}(x, t)^{\perp}\right) e^{-a x}$

$$
S(x, t)=\left(\begin{array}{c}
-e^{-2 i m x} \tanh (2 m t \sin \theta) \\
e^{2 i m x \cos \theta} \operatorname{sech}(2 m t \sin \theta)
\end{array}\right)
$$

is a wave map into $S^{3}$.

Note that if $\cos \theta=\frac{j}{2 m}$ for some integer $j$, then $s$ is periodic in $x$ with period $2 \pi$. In this case, $S$ is a wave map from $S^{1} \times \mathbb{R}$ to $S^{3}$. 


\section{Example 5.3. 1-soliton wave map into $S U(2)$}

Let $z=r+i s$, and $a, \pi$ and $s_{0}$ as in Example 5.2. We derive the formula for $\tilde{s}=g_{z, \pi} \bullet s_{0}$. A direct computation as in Example 5.2 implies that

$$
\tilde{\pi}(\xi, \eta)=\frac{1}{1+e^{2 A}}\left(\begin{array}{cc}
1 & e^{A-i B} \\
e^{A+i B} & e^{2 A}
\end{array}\right),
$$

where

$$
\begin{aligned}
& A=m s\left(x+t-\frac{x-t}{|z|^{2}}\right)=m s\left(\left(1-\frac{1}{|z|^{2}}\right) x+\left(1+\frac{1}{|z|^{2}}\right) t\right) \\
& B=m r\left(x+t+\frac{x-t}{|z|^{2}}\right)=m r\left(\left(1+\frac{1}{|z|^{2}}\right) x+\left(1-\frac{1}{|z|^{2}}\right) t\right) .
\end{aligned}
$$

The first column of $E_{0}(x, t,-1)(2 \tilde{\pi}(x, t)-\mathrm{I}) E_{0}(x, t, 1)^{-1}$ is

$$
\tilde{S}=\left(\begin{array}{c}
-e^{-2 i m x} \tanh A \\
e^{i B} \operatorname{sech} A
\end{array}\right)
$$

which is a wave map into $S^{3}$. Note that $\tilde{S}$ is periodic in $x$ if $|z|=1$ and $r$ is rational, and oscillates as $|x| \rightarrow \infty$ if $|z| \neq 1$.

Example 5.4. $k$-soliton wave map from $S^{1} \times \mathbb{R}$ to $S U(2)$

We apply Bäcklund transformations $k$ times to construct $k$-soliton wave maps from $S^{1} \times \mathbb{R}$ to $S U(2)$. Let $a, q_{0}, V$, and $\pi$ be as in Example 5.2. Let

$$
z_{j}=e^{i \theta_{j}}=\frac{r_{j}+i \mu_{j}}{m}, \quad 2 r_{j} \text { an integer, }\left|r_{j}\right|<m, \quad \mu_{j}=\sqrt{m^{2}-r_{j}^{2}}
$$

for $j=1, \cdots, k$. Set

$$
\left(a, u_{j}, v_{j}\right)=g_{z_{j}, \pi} \bullet\left(g_{z_{j-1}, \pi} \bullet \cdots \bullet g_{z_{1}, \pi} \bullet(a, 0, a) \cdots\right),
$$

$E_{j}$ the trivialization of the Lax pair of $\left(a, u_{j}, v_{j}\right)$,

$$
q_{j}(x, t)=E_{j-1}\left(x, t, z_{j}\right)^{*}\left(q_{0}\right)=E_{j-1}\left(x, t, \bar{z}_{j}\right)^{-1}\left(q_{0}\right),
$$

$\pi_{j}(x, t)$ the Hermitian projection of $\mathbb{C}^{n}$ onto $\mathbb{C} q_{j}(x, t)$, i.e.,

$$
\pi_{j}(x, t)=\frac{q_{j}(x, t) q_{j}^{*}(x, t)}{\left\|q_{j}(x, t)\right\|^{2}} .
$$

Set

$$
g_{j}(\lambda)=g_{z_{j}, \pi}(\lambda), \quad \tilde{g}_{j}(x, t, \lambda)=g_{z_{j}, \pi_{j}(x, t)}(\lambda) .
$$


By Theorem 5.1 and induction, we have

$$
\begin{gathered}
E_{j}(x, t, \lambda)=\left(g_{j} \cdots g_{1}\right)(\lambda) E_{0}(x, t, \lambda)\left(\tilde{g}_{j} \cdots \tilde{g}_{1}\right)^{-1}(x, t, \lambda), \\
q_{j}(x, t)=\left(\tilde{g}_{j-1} \cdots \tilde{g}_{1}\right)\left(\bar{z}_{j}\right) E_{0}\left(x, t, z_{j}\right)^{*}\left(g_{j-1} \cdots g_{1}\right)^{*}\left(z_{j}\right)\left(q_{0}\right),
\end{gathered}
$$

where $E_{0}(x, t, \lambda)=e^{a\left(\lambda \xi+\lambda^{-1} \eta\right)}$.

Since $\pi\left(q_{0}\right)=q_{0}, \pi^{\perp}\left(q_{0}\right)=0$,

$$
g_{i}\left(z_{j}\right)^{*}\left(q_{0}\right)=\left(\pi+\frac{\bar{z}_{j}-\bar{z}_{i}}{\bar{z}_{j}-z_{i}} \pi^{\perp}\right)\left(q_{0}\right)=q_{0} .
$$

Hence $\left(g_{j} \cdots g_{1}\right)^{*}\left(z_{j}\right)\left(q_{0}\right)=q_{0}$, and

$$
\begin{aligned}
q_{j}(x, t) & =\left(\tilde{g}_{j-1} \cdots \tilde{g}_{1}\right)\left(\bar{z}_{j}\right) E_{0}\left(x, t, z_{j}\right)^{*}\left(q_{0}\right) \\
& =\left(\tilde{g}_{j-1} \cdots \tilde{g}_{1}\right)\left(\bar{z}_{j}\right)\left(\begin{array}{c}
e^{-\left(i r_{j} x+\mu_{j} t\right)} \\
e^{i r_{j} x+\mu_{j} t}
\end{array}\right) .
\end{aligned}
$$

The wave map corresponding to $\left(a, u_{j}, v_{j}\right)$ constructed in Theorem 4.2 is

$$
s_{j}(x, t)=E_{j}(x, t,-1) E_{j}(x, t, 1)^{-1} .
$$

From Example 5.2, we get

$$
\begin{gathered}
q_{1}(x, t)=\left(\begin{array}{c}
e^{-\left(i r_{1} x+\mu_{1} t\right)} \\
e^{i r_{1} x+\mu_{1} t}
\end{array}\right), \\
\pi_{1}(x, t)=\frac{1}{e^{2 \mu_{1} t}+e^{-2 \mu_{1} t}}\left(\begin{array}{cc}
e^{-2 \mu_{1} t} & e^{-2 i r_{1} x} \\
e^{2 i r_{1} x} & e^{2 \mu_{1} t}
\end{array}\right), \\
E_{1}(x, t, \lambda)=g_{1}(\lambda) e^{a\left(\lambda \xi+\lambda^{-1} \eta\right)} \tilde{g}_{1}(x, t, \lambda)^{-1}, \\
s_{1}(x, t)=g_{1}(-1) e^{-a x}\left(\pi_{1}(x, t)-\pi_{1}^{\perp}(x, t)\right) e^{-a x} g_{1}(1)^{-1} .
\end{gathered}
$$

Since $r_{1}$ is an integer, $\pi_{1}, \tilde{g}_{1}, s_{1}$ are periodic in $x$ with period $2 \pi$.

Note that

$$
E_{0}\left(x, t, z_{j}\right)^{*}\left(q_{0}\right)=\left(\begin{array}{c}
e^{-\left(i r_{j} x+\mu_{j} t\right)} \\
e^{i r_{j} x+\mu_{j} t}
\end{array}\right) .
$$

Use (5.5b), (5.6) and (5.9) to get

$$
q_{2}(x, t)=\tilde{g}_{1}\left(x, t, \bar{z}_{2}\right)\left(\begin{array}{c}
e^{-\left(i r_{2} x+\mu_{2} t\right)} \\
e^{i r_{2} x+\mu_{2} t}
\end{array}\right) .
$$

Since $\tilde{g}_{1}$ is periodic in $x$ and $r_{2}$ is an integer, $q_{2}$ is periodic in $x$ with period $2 \pi$. So is $\pi_{2}$. The wave map corresponding to $\left(a, u_{2}, v_{2}\right)$ is

$$
s_{2}(x, t)=b_{2} e^{-a x} \tilde{g}_{1}(x, t,-1)^{-1}\left(\pi_{2}(x, t)-\pi_{2}(x, t)^{\perp}\right) \tilde{g}_{1}(x, t, 1) e^{-a x} c_{2}^{-1},
$$


where $b_{2}=\left(g_{2} g_{1}\right)(-1), c_{2}=\left(g_{2} g_{1}\right)(1)$, and $\pi_{2}(x, t)$ is the projection onto

$$
q_{2}(x, t)=\left(\pi_{1}(x, t)+\frac{\bar{z}_{1}-z_{1}}{\bar{z}_{2}-\bar{z}_{1}} \pi_{1}^{\perp}(x, t)\right)\left(\begin{array}{c}
1 \\
e^{2 i r_{2} x+\mu_{2} t}
\end{array}\right) .
$$

By assumption $2 r_{2}$ is an integer. So $q_{2}$ and $s_{2}$ are periodic in $x$ with period $2 \pi$.

It now follows from induction that

1. the wave map corresponding to $\left(a, u_{j}, v_{j}\right)$ is

$$
s_{j}(x, t)=b_{j} e^{-a x}\left(\tilde{g}_{1}^{-1} \cdots \tilde{g}_{j}^{-1}\right)(x, t,-1)\left(\tilde{g}_{j} \cdots \tilde{g}_{1}\right)(x, t, 1) c_{j}^{-1},
$$

where

$$
b_{j}=\left(g_{j} \cdots g_{1}\right)(-1), \quad c_{j}=\left(g_{j} \cdots g_{1}\right)(1),
$$

2. $s_{j}$ is periodic in $x$ with period $2 \pi$, i.e., $s_{j}$ is a wave map from $S^{1} \times \mathbb{R}$ to $S U(2)$.

Next we compute the wave map constructed by applying Bäcklund transformation to wave maps into $S^{1}$ constructed in Example 4.4.

Example 5.5. Let $z=r+i s, v_{0}=\left(\begin{array}{l}1 \\ 1\end{array}\right), h, h, a, b$ and $s$ as in Example 4.4. We use Theorem 5.1 to construct $(a, u, v)=g_{z, \pi} *(a, 0, b)$. A direct computation gives

$$
q(\xi, \eta)=E(\xi, \eta, z)^{*}\left(v_{0}\right)=\left(\begin{array}{c}
e^{-i\left(h(\xi) \bar{z}+k(\eta) \bar{z}^{-1}\right)} \\
e^{i\left(h(\xi) \bar{z}+k(\eta) \bar{z}^{-1}\right)}
\end{array}\right),
$$

which is parallel to

$$
\left(\begin{array}{c}
1 \\
e^{2 s\left(h(\xi)-\frac{k(\eta)}{r^{2}+s^{2}}\right)} e^{2 i r\left(h(\xi)+\frac{k(\eta)}{r^{2}+s^{2}}\right)}
\end{array}\right) .
$$

So the projection $\tilde{\pi}(\xi, \eta)$ onto $\mathbb{C} q(\xi, \eta)$ is

$$
\tilde{\pi}(\xi, \eta)=\frac{1}{1+e^{4 s\left(h(\xi)-\frac{k(\eta)}{r^{2}+s^{2}}\right)}}\left(\begin{array}{cc}
1 & \bar{f} \\
f & |f|^{2}
\end{array}\right),
$$

where $f=e^{2 s\left(h(\xi)-\frac{k(\eta)}{r^{2}+s^{2}}\right)+2 i r\left(h(\xi)+\frac{k(\eta)}{r^{2}+s^{2}}\right)}$. Then

$$
\begin{aligned}
& u=2 i s[\tilde{\pi}, a] \\
& v=\frac{1}{|z|^{2}}\left(\bar{z} \tilde{\pi}+z \tilde{\pi}^{\perp}\right) b(\eta)\left(z \tilde{\pi}+\bar{z} \tilde{\pi}^{\perp}\right) .
\end{aligned}
$$


The wave map corresponding to $(a, u, v)$ is

$$
\tilde{s}=g_{z, \pi} * s=g_{z, \pi}(-1) e^{-a x}\left(\tilde{\pi}(x, t)-\tilde{\pi}(x, t)^{\perp}\right) e^{-a x} g_{z, \pi}(-1) .
$$

The first column $S$ of $e^{-a x}\left(\tilde{\pi}(x, t)-\tilde{\pi}(x, t)^{\perp}\right) e^{-a x}$ is

$$
S(x, t)=\left(\begin{array}{c}
-e^{-2 i m x} \tanh A \\
e^{i B} \operatorname{sech} A
\end{array}\right)
$$

where

$$
\begin{aligned}
& A=2 s\left(h\left(\frac{x+t}{2}\right)-\frac{1}{r^{2}+s^{2}} k\left(\frac{x-t}{2}\right)\right), \\
& B=2 r\left(h\left(\frac{x+t}{2}\right)+\frac{1}{r^{2}+s^{2}} k\left(\frac{x-t}{2}\right)\right) .
\end{aligned}
$$

If $h(x)$ and $k(x)$ tends to zero as $|x| \rightarrow \infty$, then $\lim _{|x| \rightarrow \infty} \tilde{\pi}(x, t)=\frac{1}{2}\left(\begin{array}{ll}1 & 1 \\ 1 & 1\end{array}\right)$. In this case the wave maps $\tilde{s}$ and $S$ have constant boundary condition.

We claim that if $h^{\prime}$ and $k^{\prime}$ are square integrable then the wave map $\tilde{s}$ has finite energy at each level $t$. This can be proved using Theorem 4.2. The energy at $t=t_{0}$ is

$$
\int_{t=t_{0}}\left\|\tilde{s}^{-1} \tilde{s}_{x}\right\|^{2} d x=\int_{t=t_{0}} \frac{1}{2}\left\|\tilde{s}^{-1} \tilde{s}_{\xi}+\tilde{s}^{-1} \tilde{s}_{\eta}\right\|^{2} d x .
$$

Recall that the trivialization $\tilde{E}$ of the Lax pair of $(a, u, v)$ satisfies the reality condition (2.7). So $\tilde{E}(x, t, \pm 1) \in U(2)$. Thus by Theorem 4.2 , we have

$$
\left\|\tilde{s}^{-1} \tilde{s}_{\xi}\right\|=2\left\|h^{\prime}\right\|, \quad\left\|\tilde{s}^{-1} \tilde{s}_{\eta}\right\|=2\|v\| .
$$

Since $\frac{1}{|z|}\left(\bar{z} \tilde{\pi}+z \tilde{\pi}^{\perp}\right) \in U(2)$,

$$
\|v(\xi, \eta)\|=\left\|k^{\prime}(\eta)\right\|
$$

The assumption $h^{\prime}, k^{\prime}$ are in $L^{2}$ implies that

$$
\int_{t=t_{0}}\left\|\tilde{s}^{-1} \tilde{s}_{x}\right\|^{2} d x<\infty
$$




\section{Homoclinic wave maps.}

We study the asymptotic behavior of the periodic $k$-soliton wave maps $s_{j}$ constructed in Example 5.4, and prove that they are homoclinic.

We use the same notations as in Example 5.4. First we look at the behavior of $s_{j}$ as $t \rightarrow-\infty$. Set

$$
y_{j}=e^{2 \mu_{j} t}, \quad f_{j}=e^{2 i r_{j} x} .
$$

Since $\mu_{j}>0, \lim _{t \rightarrow-\infty} y_{j}=0$. By $(5.8 \mathrm{a}), q_{1}(x, t)$ is parallel to

$$
\hat{q}_{1}=\left(\begin{array}{c}
1 \\
f_{1} y_{1}
\end{array}\right)=\left(\begin{array}{l}
1 \\
0
\end{array}\right)+f_{1} y_{1}\left(\begin{array}{l}
0 \\
1
\end{array}\right) .
$$

So the projection $\pi_{1}(x, t)$ onto $\mathbb{C} \hat{q}_{1}(x, t)$ is

$$
\pi_{1}(x, t)=\left(\begin{array}{ll}
1 & 0 \\
0 & 0
\end{array}\right)+y_{1}\left(\begin{array}{cc}
0 & \bar{f}_{1} \\
f_{1} & 0
\end{array}\right)+O\left(y_{1}^{2}\right)
$$

where $O\left(y_{1}^{2}\right)$ means terms involving $y_{1}^{n}$ with $n \geq 2$. By $(5.7), q_{2}(x, t)$ is parallel to

$$
\hat{q}_{2}(x, t)=\tilde{g}_{1}\left(\bar{z}_{2}\right)\left(\begin{array}{c}
1 \\
f_{2} y_{2}
\end{array}\right) .
$$

Expand it in $y_{1}, y_{2}$ to get

$$
\hat{q}_{2}(x, t)=\left(\begin{array}{l}
1 \\
0
\end{array}\right)+\left(c_{1} f_{1} y_{1}+c_{2} f_{2} y_{2}\right)\left(\begin{array}{l}
0 \\
1
\end{array}\right)+O\left(y^{2}\right),
$$

where $c_{1}, c_{2}$ are constants depending on $z_{1}, z_{2}$. This implies that the projection

$$
\pi_{2}(x, t)=\left(\begin{array}{ll}
1 & 0 \\
0 & 0
\end{array}\right)+y_{1}\left(\begin{array}{cc}
0 & \bar{\alpha}_{1} \bar{f}_{1} \\
\alpha_{1} f_{1} & 0
\end{array}\right)+y_{2}\left(\begin{array}{cc}
0 & \bar{\alpha}_{2} \bar{f}_{2} \\
\alpha_{2} f_{2} & 0
\end{array}\right)+O\left(y^{2}\right),
$$

where $\alpha_{1}, \alpha_{2}$ are constants depending on $z_{1}, z_{2}$. Use the formula for $q_{j}, \pi_{j}, E_{j}$ and induction to see that as $t \rightarrow-\infty$ we have

$$
\pi_{j}(x, t)=\left(\begin{array}{ll}
1 & 0 \\
0 & 0
\end{array}\right)+\sum_{r=1}^{j} y_{r}\left(\begin{array}{cc}
0 & \bar{\alpha}_{r} \bar{f}_{r} \\
\alpha_{r} f_{r} & 0
\end{array}\right)+O\left(y^{2}\right)
$$

where $\alpha_{r}$ are constants depending on $z_{1}, \ldots, z_{j}$. In particular, this proves that

$$
\lim _{t \rightarrow-\infty} \pi_{j}(x, t)=\left(\begin{array}{ll}
1 & 0 \\
0 & 0
\end{array}\right)
$$


Next we use formula (5.10) to compute the asymptotic behavior of $s_{j}$ as $t \rightarrow-\infty$. It follows from (6.2) that we have

$$
\lim _{t \rightarrow-\infty} \tilde{g}_{r}(x, t,-1)^{-1} \tilde{g}_{r}(x, t, 1)=\left(\begin{array}{cc}
1 & 0 \\
0 & \frac{1+\bar{z}_{r}}{1+z_{r}}
\end{array}\right)\left(\begin{array}{cc}
1 & 0 \\
0 & \frac{1-z_{r}}{1-\bar{z}_{r}}
\end{array}\right)=\left(\begin{array}{cc}
1 & 0 \\
0 & -1
\end{array}\right) .
$$

By (5.10), we have

$$
\lim _{t \rightarrow-\infty} s_{j}(x, t)=b_{j} e^{-2 a x}\left(\begin{array}{cc}
1 & 0 \\
0 & -1
\end{array}\right)^{j} c_{j}^{-1}
$$

where $b_{j}, c_{j}$ are given by (5.11). In particular, we get

$$
\begin{gathered}
\lim _{t \rightarrow-\infty} s_{2 k}(x, t)=b_{2 k} e^{-2 a x} c_{2 k}^{-1}, \\
\lim _{t \rightarrow-\infty} s_{2 k+1}(x, t)=b_{2 k+1} e^{-2 a x}\left(\begin{array}{cc}
1 & 0 \\
0 & -1
\end{array}\right) c_{2 k+1}^{-1} .
\end{gathered}
$$

Both $b_{j}$ and $c_{j}$ lies in $U(2)$. A direct computation gives

$$
g_{z, \pi}(-1) g_{z, \pi}(1)^{-1}=\pi-\pi^{\perp} .
$$

This implies $b_{2 k} c_{2 k}^{-1} \in S U(2)$. So

$$
\hat{s}_{2 k}=b_{2 k}^{-1} s_{2 k} c_{2 k}
$$

is a wave map from $S^{1} \times \mathbb{R}^{1}$ to $S U(2)$. Use (5.10) and (6.1) and a direct computation to conclude

$$
\hat{s}_{2 k}(x, t)=e^{-2 a x}+e^{-a x}\left(\sum_{j=1}^{2 k} y_{j}\left(\begin{array}{cc}
0 & \bar{\beta}_{j} \bar{f}_{j} \\
-\beta_{j} f_{j} & 0
\end{array}\right)\right) e^{-a x}+O\left(y^{2}\right)
$$

as $t \rightarrow-\infty$, where $\beta_{1}, \ldots, \beta_{2 k}$ are real constants. Recall that

$$
\begin{aligned}
& s_{0}(x, t)=e^{-2 a x}=\operatorname{diag}\left(e^{-2 i m x}, e^{2 i m x}\right) . \\
& s_{0}^{-1}(x, t) y_{j} e^{-a x}\left(\begin{array}{cc}
0 & \bar{\beta}_{j} \bar{f}_{j} \\
-\beta_{j} f_{j} & 0
\end{array}\right) e^{-a x} \\
& =e^{2 \mu_{j} t}\left(\begin{array}{cc}
0 & \bar{\beta}_{j} e^{2 i\left(m-r_{j}\right) x} \\
-\beta_{j} e^{-2 i\left(m-r_{j}\right) x} & 0
\end{array}\right),
\end{aligned}
$$


which is an unstable mode $p_{-2 m, 2 r_{j}}^{+}$at the stationary solution $s_{0}$ with eigenvalue $2 \sqrt{m^{2}-r_{j}^{2}}=2 \mu_{j}$ as given in Corollary 3.3. In other words, we have shown

$$
\lim _{t \rightarrow-\infty} \hat{s}_{2 k}(x, t)-s_{0}(x, t)-s_{0}(x, t) \sum_{j=1}^{2 k} p_{-2 m, 2 r_{j}}^{+}(x, t)=0 .
$$

To compute the asymptotic behavior of $s_{j}(x, t)$ as $t \rightarrow \infty$, we set

$$
h_{j}=e^{-2 i r_{j} x}, \quad \rho_{j}=e^{-2 \mu_{j} t} .
$$

Since $\mu_{j}>0, \lim _{t \rightarrow \infty} \rho_{j}=0$. A similar computation implies that

1. $q_{1}(x, t)$ is parallel to $\left(\begin{array}{c}h_{1} \rho_{1} \\ 1\end{array}\right)$,

2.

$$
\pi_{j}(x, t)=\left(\begin{array}{ll}
0 & 0 \\
0 & 1
\end{array}\right)+\sum_{n=1}^{j} \rho_{n}\left(\begin{array}{cc}
0 & \bar{\beta}_{n}^{\prime} \bar{h}_{n} \\
\beta_{n}^{\prime} h_{n} & 0
\end{array}\right)+O\left(\rho^{2}\right)
$$

for some constants $\beta_{1}^{\prime}, \ldots, \beta_{j}^{\prime}$ depending on $z_{1}, \cdots, z_{j}$,

3. $\lim _{t \rightarrow \infty} \tilde{g}_{j}(x, t,-1)^{-1} \tilde{g}_{j}(x, t, 1)=\left(\begin{array}{cc}-1 & 0 \\ 0 & 1\end{array}\right)$,

4.

$$
\begin{gathered}
\lim _{t \rightarrow \infty} s_{2 k}(x, t)=b_{2 k} e^{-2 a x} c_{2 k}^{-1}, \\
\lim _{t \rightarrow \infty} s_{2 k+1}(x, t)=b_{2 k+1} e^{-2 a x}\left(\begin{array}{cc}
-1 & 0 \\
0 & 1
\end{array}\right) c_{2 k+1}^{-1} .
\end{gathered}
$$

5.

$\hat{s}_{2 k}(x, t)=e^{-2 a x}+e^{-a x} \sum_{j=1}^{2 r} \rho_{j}\left(\begin{array}{cc}0 & \bar{\epsilon}_{j} \bar{h}_{j} \\ -\epsilon_{j} h_{j} & 0\end{array}\right) e^{-a x}+O\left(\rho^{2}\right)$, as $t \rightarrow \infty$ for some constants $\epsilon_{1}, \ldots, \epsilon_{j}$ depending on $z_{1}, \cdots, z_{j}$.

6. As $t \rightarrow \infty$ we have

$$
\lim _{t \rightarrow \infty} \hat{s}_{2 k}(x, t)-s_{0}(x, t)-s_{0}(x, t) \sum_{j=1}^{2 k} p_{-2 m, 2 r_{j}}^{-}(x, t)=0
$$

for some linear stable mode $p_{-2 m, 2 r_{j}}^{-}$. 
As a consequence of $(6.3 \mathrm{~b})$ and $(6.6 \mathrm{~b})$, we get

$$
\lim _{t \rightarrow-\infty} s_{2 k+1}(x, t)=-\lim _{t \rightarrow \infty} s_{2 k+1}(x, t)=b_{2 k+1} e^{-2 a x}\left(\begin{array}{cc}
1 & 0 \\
0 & -1
\end{array}\right) c_{2 k+1}^{-1} .
$$

So $s_{2 k+1}$ is a hecteroclinic wave map from $S^{1} \times \mathbb{R}^{1}$ to $S U(2)$.

Formulas (6.4) and (6.7) imply that $s_{2 k}, \hat{s}_{2 k}$ are homoclinic wave map from $S^{1} \times \mathbb{R}^{1}$ to $S U(2)$. So we have

Theorem 6.1. Let $2 m$ be an integer, $a=\operatorname{diag}(i m,-i m)$, and $s_{0}(x, t)=$ $e^{-2 a x}$. Let $\pi$ denote the projection of $\mathbb{C}^{2}$ onto $\mathbb{C}\left(\begin{array}{l}1 \\ 1\end{array}\right)$, and

$$
z_{j}=e^{i \theta_{j}}=\frac{r_{j}}{m}+i \frac{\sqrt{m^{2}-r_{j}^{2}}}{m}=\frac{r_{j}+i \mu_{j}}{m},
$$

where $2 r_{1}, \ldots, 2 r_{2 k}$ are integers and $\left|r_{j}\right|<m$. Let

$$
s_{2 k}=g_{z_{2 k}, \pi} \bullet\left(\cdots \bullet\left(g_{z_{1}, \pi} \bullet s_{0}\right) \cdots\right)
$$

denote the wave map obtained by applying $2 k$ Bäcklund transformations. Then $s_{2 k}$ is a homoclinic wave map from $S^{1} \times \mathbb{R}^{1}$ to $S U(2)$. Moreover, there exist constants $b_{2 k}, c_{2 k} \in U(2)$ such that $\hat{s}_{2 k}=b_{2 k}^{-1} s_{2 k} c_{2 k}$ satisfies $\lim _{|t| \rightarrow \infty} s_{2 k}(x, t)=s_{0}(x, t)$ and (6.4) and (6.7).

\section{7. wave maps into $S^{2}$.}

We describe a constraint condition for solutions the -1 equation associated to $S U(2)=S^{3}$ so that the corresponding wave maps into $S^{3}$ actually lies in $S^{2}$.

Recall that we identify $S^{3} \subset \mathbb{C}^{2}=\mathbb{R}^{4}$ as $S U(2)$ via

$$
\left(\begin{array}{c}
z \\
w
\end{array}\right) \mapsto\left(\begin{array}{cc}
z & -\bar{w} \\
w & \bar{z}
\end{array}\right)
$$

The intersection of $S^{3}$ with the linear hyperplane defined by $\operatorname{Re}(w)=0$ is

$$
M=\left\{\left.\left(\begin{array}{cc}
z & i s \\
i s & \bar{z}
\end{array}\right)|z \in \mathbb{C}, s \in \mathbb{R},| z\right|^{2}+s^{2}=1\right\},
$$

which is a totally geodesic 2 -sphere in $S U(2)=S^{3}$. If $y \in S U(2)$, then $y \in M$ if and only if $y^{t}=y$. 
It is well-known that if $s$ is a wave map into a Riemannian manifold $N$ and the image of $s$ lies in a totally geodesic submanifold $M$ of $N$, then $s$ is a wave map into $M$. Hence if a wave map into $S^{3}$ with its image lies in a totally geodesic $S^{2}$, then this is also a wave map into $S^{2}$.

In this section, we give a constraint condition on solutions of the -1 flow equation so that the corresponding wave maps lie in the totally geodesic $S^{2}$ in $S U(2)=S^{3}$. As a consequence, we also see that the SGE is a subequation of the constrained -1 flow equation.

In order to explain how to get wave maps into $S^{2}$, we need to view $S^{2}$ as the symmetric space $S U(2) / S O(2)$. Let $\tau, \sigma: S L(2, \mathbb{C}) \rightarrow S L(2, \mathbb{C})$ denote the maps defined by

$$
\tau(g)=\left(g^{*}\right)^{-1}, \quad \sigma(g)=\left(g^{t}\right)^{-1} .
$$

Then $\sigma$ and $\tau$ are group homomorphisms, $\sigma^{2}=\tau^{2}=\mathrm{id}, \sigma \tau=\tau \sigma$, and the differentials at the identity matrix are

$$
\sigma_{*}(\xi)=-\xi^{t}, \quad \tau_{*}(\xi)=-\xi^{*} .
$$

Note that $\sigma(S U(2)) \subset S U(2)$. Hence $\sigma \mid S U(2)$ is an involution of $S U(2)$. The fixed point set of $\sigma$ in $S U(2)$ is $S O(2)$, and $S^{2}$ is diffeomorphic to $S U(2) / S O(2)$. A direct computation shows that

$$
M_{\sigma}=\left\{g \sigma(g)^{-1} \mid g \in S U(2)\right\}
$$

is the totally geodesic $M=S^{2}$ given in (7.1).

Since $\sigma_{*}^{2}=$ id, we have:

$$
s u(2)=\mathcal{K}+\mathcal{P},
$$

where $\mathcal{K}$ and $\mathcal{P}$ are eigenspaces of $\sigma_{*}$ on $s u(2)$ with eigenvalues $1,-1$ respectively. In fact,

$$
\mathcal{K}=s o(2), \quad \mathcal{P}=\left\{i\left(\begin{array}{ll}
x & y \\
y & x
\end{array}\right) \mid x, y \in \mathbb{R}\right\} .
$$

Since $\sigma_{*}$ is a Lie algebra automorphism, $\sigma_{*}\left(\left[\eta_{1}, \eta_{2}\right]\right)=\left[\sigma_{*}\left(\eta_{1}\right), \sigma_{*}\left(\eta_{2}\right)\right]$. So we have

$$
[\mathcal{K}, \mathcal{K}] \subset \mathcal{K}, \quad[\mathcal{K}, \mathcal{P}] \subset \mathcal{P}, \quad[\mathcal{P}, \mathcal{P}] \subset \mathcal{K} .
$$

Proposition 7.1. Let $(a, u, v)$ be a solution of the -1 flow equation associated to $S U(2), \theta_{\lambda}=(a \lambda+u) d \xi+\lambda^{-1} d \eta$ its Lax pair, and $E$ the trivialization of $\theta_{\lambda}$. Then the following statements are equivalent: 
1. $a, v \in \mathcal{P}$ and $u \in \mathcal{K}$.

2. $\theta_{\lambda}$ satisfies

$$
\theta_{\lambda}=-\theta_{\bar{\lambda}}^{*}, \quad \theta_{\lambda}=-\theta_{-\lambda}^{t} .
$$

3. The trivialization $E(x, t, \lambda)$ of $\theta_{\lambda}$ satisfies

$$
E(x, t, \bar{\lambda})^{*} E(x, t, \lambda)=\mathrm{I}, \quad E(x, t, \lambda) E(x, t,-\lambda)^{t}=\mathrm{I},
$$

Proof. It is easy to see that (1) and (2) are equivalent and (3) implies (2). To prove (2) implies $(3)$, we let $F(x, t, \lambda)=\left(E(x, t,-\lambda)^{t}\right)^{-1}$. Then

$$
F^{-1} d F=-\theta_{-\lambda}^{t}=\theta_{\lambda}, \quad F(0,0, \lambda)=\mathrm{I}
$$

Hence $E=F$, i.e., $E(x, t, \lambda) E(x, t,-\lambda)^{t}=\mathrm{I}$. We have shown before that $\theta_{\lambda}=-\theta_{\bar{\lambda}}^{*}$ implies that $E(x, t, \bar{\lambda})^{*} E(x, t, \lambda)=\mathrm{I}$.

Proposition 7.2. Let $(a, u, v)$ be a solution of the -1 flow equation associated to $S U(2)$, and $s$ the wave map constructed from $(a, u, v)$ in Theorem 4.2. If $a, v \in \mathcal{P}$ and $u \in \mathcal{K}$, then $s$ is a wave map into $S^{2}$.

Proof. By Proposition 7.1, the trivialization $E$ of the Lax pair corresponding to $(a, u, v)$ satisfies the reality condition (7.4). This implies that $E(x, t, r) \in S U(2)$ for all $r \in \mathbb{R}$ and $E(x, t, 1)^{-1}=E(x, t,-1)^{t}$. The wave map constructed in Theorem 4.2 is

$$
s(x, t)=E(x, t,-1) E(x, t, 1)^{-1}=E(x, t,-1) E(x, t,-1)^{t} .
$$

But $y \in S U(2)$ lies in $S^{2}$ given in (7.1) if and only if $y^{t}=y$. So $s(x, t)$ lies in $S^{2}$. Because $S^{2}$ is totally geodesic in $S U(2), s$ is a wave map into $S^{2}$.

The following Proposition was proved in [5].

Proposition 7.3. Let $(a, u, v)$ be a solution of the -1 flow equation associated to $S U(2)$ and $a, v \in \mathcal{P}$ and $u \in \mathcal{K}$, and $s$ the wave map corresponding to $(a, u, v)$ in Theorem 4.2. Then:

(1) If $z=i \mu$ is pure imaginary and $\bar{\pi}=\pi$, then $(a, \tilde{u}, \tilde{v})=g_{i \mu, \pi} \bullet(a, u, v)$ is again a solution of the -1 flow with $\tilde{u} \in \mathcal{K}, \tilde{v} \in \mathcal{P}$, and $g_{i \mu, \pi} \bullet s$ is a wave map into $S^{2}$. 
(2) If $\bar{\pi}=\pi$, then $(a, \hat{u}, \hat{v})=g_{z, \pi} \bullet\left(g_{-\bar{z}, \pi} \bullet(a, u, v)\right)$ is a solution of the -1 flow with $\hat{u} \in \mathcal{K}, \hat{v} \in \mathcal{P}$, and $g_{z, \pi} \bullet\left(g_{-\bar{z}, \pi} \bullet s\right)$ is a wave map into $S^{2}$.

Example 7.4. SGE and wave maps into $S^{2}([2,3,5])$.

Let $s u(2)=\mathcal{K}+\mathcal{P}$, where $\mathcal{K}$ and $\mathcal{P}$ are given by (7.2). Let

$$
a=\operatorname{diag}(i,-i), \quad u=\left(\begin{array}{cc}
0 & \frac{q_{\xi}}{2} \\
-\frac{q_{\xi}}{2} & 0
\end{array}\right), \quad v=-\frac{i}{4}\left(\begin{array}{cc}
\cos q & \sin q \\
\sin q & -\cos q
\end{array}\right) .
$$

Note $a, v \in \mathcal{P}$ and $u \in \mathcal{K}$. A direct computation implies that:

(1) $(a, u, v)$ is a solution of the -1 flow equation associated to $S U(2)$ if and only if $q$ is a solution of the sine-Gordon equation (SGE):

$$
q_{\xi \eta}=\sin q .
$$

Hence solutions of the SGE give rise to wave maps into $S^{2}$.

(2) Let $(a, \tilde{u}, \tilde{v})=g_{i s, \pi} \bullet(a, u, v)$. If $\bar{\pi}=\pi$, then

$$
\tilde{u}=\left(\begin{array}{cc}
0 & \frac{\tilde{q}_{\xi}}{2} \\
-\frac{\tilde{q}_{\xi}}{2} & 0
\end{array}\right), \quad \tilde{v}=-\frac{i}{4}\left(\begin{array}{cc}
\cos \tilde{q} & \sin \tilde{q} \\
\sin \tilde{q} & -\cos \tilde{q}
\end{array}\right)
$$

for some $\tilde{q}$. So $\tilde{q}$ is again a solution of the SGE. Let $g_{i s, \pi} \bullet q$ denote $\tilde{q}$.

(3) $g_{i s, \pi} \bullet 0$ is the traveling wave solution of the SGE:

$$
q(\xi, \eta)=4 \tan ^{-1}\left(e^{s \xi+\frac{\eta}{s}}\right),
$$

and $g_{e^{i \theta}, \pi} \bullet\left(g_{-e^{-i \theta}, \pi} \bullet 0\right)$ is the breather solution of the SGE:

$$
4 \tan ^{-1}\left(\frac{\sin \theta \sin (t \cos \theta)}{\cos \theta \cosh (x \sin \theta)}\right)
$$

where $x, t$ are space and time coordinates.

This solution is periodic in time, and hence called a breather. But wave map equation is invariant if we exchange the space and time variables. So a breather solution can also be viewed as periodic in space instead.

Since wave maps into $S^{2}$ is a special cases of wave maps into $S U(2)$, as a consequence of Theorem 6.1 and Proposition 7.3 we get the following Theorem:

Theorem 7.5. We use the same assumption as in Theorem 6.1. If $z_{2 j}=$ $-\bar{z}_{2 j-1}$, i.e., $r_{2 j}=-r_{2 j-1}$ and $\mu_{2 j}=\mu_{2 j-1}$ for all $1 \leq j \leq k$, then $s_{2 k}$ is a homoclinic $2 k$-soliton wave map from $S^{1} \times \mathbb{R}^{1}$ into $S^{2}$.

When $k=1$, the above Theorem was proved by Shatah and Strauss in [3]. 


\section{Wave maps into compact symmetric spaces.}

In section 7, we embed $S^{2}$ as a totally geodesic submanifold of $S U(2)=S^{3}$. By viewing $S^{2}$ as the symmetric space $S U(2) / S O(2)$, we give conditions on solutions of the -1 flow equation whose image lies in $S^{2}$. In fact, this same method works for any compact symmetric space. In particular, we apply this method to construct homoclinic periodic $2 k$-soliton wave maps into $\mathbb{C} P^{n}$ and $4 k$-soliton wave maps into $S^{n}$.

First we give a short review of symmetric spaces. Let $G$ be a complex semi-simple Lie group, and $\tau$ and $\sigma$ involutions of $G$ such that

(i) the differential $\tau_{*}=d \tau_{e}$ and $\sigma_{*}=d \sigma_{e}$ at the identity $e$ are conjugate linear and complex linear Lie algebra involution on $\mathcal{G}$ respectively, i.e., $\tau_{*}(\alpha \xi)=\bar{\alpha} \tau_{*}(\xi)$ and $\sigma_{*}(\alpha \xi)=\alpha \sigma_{*}(\xi)$ for all $\alpha \in \mathbb{C}$ and $\xi \in \mathcal{G}$,

(ii) $\sigma \tau=\tau \sigma$.

Let $U$ denote the fixed point set of $\tau$ in $G$. Such $U$ is called a real form of $G$. Since $\tau$ and $\sigma$ commute, $\sigma(U) \subset U$. Let $K$ denote the fixed point set of $\sigma$ in $U$, and $\mathcal{P}$ the -1 eigenspace of $\sigma_{*}$ on $\mathcal{U}$. Then $U / K$ is a symmetric space, and $\mathcal{U}=\mathcal{K}+\mathcal{P}$ satisfying

$$
[\mathcal{K}, \mathcal{K}] \subset \mathcal{K}, \quad[\mathcal{K}, \mathcal{P}] \subset \mathcal{P}, \quad[\mathcal{P}, \mathcal{P}] \subset \mathcal{K}
$$

Let $D$ be a domain in $\mathbb{C}$ that is invariant under complex conjugation. We say that $g: D \rightarrow G$ and $\xi: D \rightarrow \mathcal{G}$ satisfy the $U$-reality condition if

$$
\tau(g(\bar{\lambda}))=g(\lambda), \quad \tau_{*}(\xi(\bar{\lambda}))=\xi(\lambda),
$$

and satisfy the $U / K$-reality condition if

$$
\left\{\begin{array}{l}
\tau(g(\bar{\lambda}))=g(\lambda), \quad \sigma(g(-\lambda))=g(\lambda) \\
\tau_{*}(\xi(\bar{\lambda}))=\xi(\lambda), \quad \sigma_{*}(\xi(-\lambda))=\xi(\lambda)
\end{array}\right.
$$

respectively.

Let $*$ denote the $U$-action on $U$ defined by $g * h=g h \sigma(g)^{-1}$. Then the stablizer at the identity $e$ is $K$. So the orbit at $e$,

$$
M_{\sigma}=\left\{g \sigma(g)^{-1} \mid g \in U\right\},
$$

is diffeomorphic to $U / K$. It is known that $M_{\sigma}$ is isometric to the symmetric space $U / K$ (for a proof see [4]): 
Proposition 8.1. The $U$-orbit $M_{\sigma}=U * e$ is totally geodesic submanifold of $U$ and is an isometric embedding of the symmetric space $U / K$ into $U$.

The embedding of $U / K$ given in the above Proposition is called the Cartan embedding. The embedding of $S^{2}$ in $S U(2)=S^{3}$ given by (7.1) is the Cartan embedding of $M_{\sigma}$ in $S U(2)$, where $\frac{S U(2)}{S O(2)}=S^{2}$ is the symmetric space given by $\tau(g)=\left(g^{*}\right)^{-1}$ and $\sigma(g)=\left(g^{t}\right)^{-1}$.

The -1 flow equation associated to $U$ is the equation (4.1) for $(a, u, v)$ : $\mathbb{R}^{2} \rightarrow \prod_{i=1}^{3} \mathcal{U}$, and has a Lax pair $\theta_{\lambda}=(a \lambda+u) d x+\lambda^{-1} v d t$. Our computations and results for $S U(2)$ and $S^{2}=\frac{S U(2)}{S O(2)}$ in previous sections work for any compact Lie group $U$ and symmetric space $U / K$. For example, the following can be proved in a similar manner:

1. The Lax pair $\theta_{\lambda}$ of the -1 flow equation associated to $U$ satisfies the $U$-reality condition (8.1), i.e.,

$$
\tau_{*}\left(\theta_{\bar{\lambda}}\right)=\theta_{\lambda}
$$

2. Theorem 4.2 holds by replacing $S U(n)$ by $U$. In other words, we have a correspondence between solutions of the -1 flow equation associated to $U$ and wave maps into $U$.

3. Since any compact Lie group $U$ can be embedded as a subgroup of $S U(N)$ for some $N$, to construct explicit solutions of the -1 flow equation, we only need to find product of simple elements (i.e., of the form (5.2)) that satisfies the $U$-reality condition.

4. The linearization of the wave map equation from $S^{1} \times \mathbb{R}^{1}$ into $U$ at the stationary wave map $s_{0}(x, t)=e^{a x}$ with $e^{2 \pi a}=\mathrm{I}$ is

$$
\xi_{t t}=\xi_{x x}+\left[a, \xi_{x}\right]
$$

Its stable and unstable modes can be computed using roots of $\mathcal{U}$.

5. Propositions 7.1 and 7.2 hold if we replace $S U(2)$ and $\frac{S U(2)}{S O(2)}$ by $U$ and $\frac{U}{K}$. The proofs are similar.

6. There are analogous Theorem 6.1 and Theorem 7.5 for $U$ and $U / K$.

Next we give two examples: 
Example 8.2. Wave maps from $S^{1} \times \mathbb{R}^{1}$ to $\mathbb{C} P^{n-1}$

Let $G=S L(n, \mathbb{C}), J=\operatorname{diag}(1, \ldots, 1,-1)$, and $\tau, \sigma: G \rightarrow G$ defined by

$$
\tau(y)=\left(y^{*}\right)^{-1}, \quad \sigma(y)=J y J^{-1} .
$$

A direct computation shows that both $\tau$ and $\sigma$ are group homomorphisms, $\tau^{2}=\sigma^{2}=\mathrm{Id}$, and $\tau \sigma=\sigma \tau$. The fixed point set of $\tau$ in $G$ is $U=S U(n)$, and the fixed point set of $\sigma$ in $U$ is $S(U(1) \times U(n-1))$. The \pm 1 eigenspace of $\sigma_{*}$ in $\mathcal{U}$ is

$$
\begin{aligned}
& \mathcal{K}=\left\{\left(\begin{array}{cc}
\xi & 0 \\
0 & c
\end{array}\right) \mid \xi \in u(n-1), c \in \mathbb{C} \text { pure imaginary, } \operatorname{tr}(\xi)+c=0\right\} \\
& \mathcal{P}=\left\{\left(\begin{array}{cc}
0 & \bar{v}^{t} \\
-v & 0
\end{array}\right) \mid v \in \mathcal{M}_{1 \times(n-1)}(\mathbb{C})\right\} .
\end{aligned}
$$

Here $\mathcal{M}_{k \times j}(K)$ is the space of $k \times j$ matrices with entries in $K$. The symmetric space corresponding to $\tau, \sigma$ is $\frac{S U(n)}{S(U(n-1) \times O(1))}=\mathbb{C} P^{n-1}$.

Let $D \in g l(n-1, \mathbb{C}), v \in \mathcal{M}_{(n-1) \times 1}(\mathbb{C}), v \in \mathcal{M}_{1 \times(n-1)}(\mathbb{C}), c \in \mathbb{C}$. Then $g=\left(\begin{array}{ll}D & b \\ v & c\end{array}\right) \in S U(n)$ if and only if

$$
\left\{\begin{array}{l}
D \bar{D}^{t}+b \bar{b}^{t}=\mathrm{I}, \\
v \bar{D}^{t}+c \bar{b}^{t}=0, \\
\|v\|^{2}+|c|^{2}=1 .
\end{array}\right.
$$

So

$$
g \sigma(g)^{-1}=\left(\begin{array}{cc}
I-2 b \bar{b}^{t} & 2 b \bar{c} \\
-2 c \bar{b}^{t} & 2|c|^{2}-1
\end{array}\right) .
$$

The map from the symmetric space $M_{\sigma}$ to $\mathbb{C} P^{n-1}$ (the space of complex linear lines in $\mathbb{C}^{n}$ ) defined by

$$
\left(\begin{array}{cc}
I-2 b \bar{b}^{t} & 2 b \bar{c} \\
-2 c \bar{b}^{t} & 2|c|^{2}-1
\end{array}\right) \mapsto \mathbb{C}\left(\begin{array}{c}
2 b \bar{c} \\
2|c|^{2}-1
\end{array}\right)
$$

is an isometry from $M_{\sigma}$ to $\mathbb{C} P^{n-1}$.

In order to construct explicit wave maps into $\mathbb{C} P^{n-1}$, we look for a product of simple elements,

$$
g=g_{z_{1}, \pi_{1}} \cdots g_{z_{r}, \pi_{r}},
$$

that satisfies the extra reality condition

$$
g(-\lambda)=\sigma(g(\lambda)) .
$$


It can be checked that the simple element $g_{z, \pi}$ does not satisfy this extra reality condition. But we can find product of two simple elements do. Let $\pi$ denote the Hermitian projection onto $\mathbb{C}\left(\begin{array}{l}w \\ c\end{array}\right)$, where $w \in \mathbb{C}^{n-1}$ and $c \in \mathbb{C}$ so that $\|w\|^{2}=|c|^{2}=1$. A direct computation implies

$$
\begin{gathered}
\pi=\frac{1}{2}\left(\begin{array}{cc}
w \bar{w}^{t} & w \bar{c} \\
c \bar{w}^{t} & |c|^{2}
\end{array}\right), \\
\sigma(\pi)=\frac{1}{2}\left(\begin{array}{cc}
w \bar{w}^{t} & -w \bar{c} \\
-c \bar{w}^{t} & |c|^{2}
\end{array}\right)=\text { projection onto } \mathbb{C}\left(\begin{array}{c}
w \\
-c
\end{array}\right) .
\end{gathered}
$$

Since $\left(\begin{array}{c}w \\ c\end{array}\right)$ and $\left(\begin{array}{c}w \\ -c\end{array}\right)$ are perpendicular with respect to the Hermitian inner product, we have

$$
\pi \sigma(\pi)=\sigma(\pi) \pi=0
$$

So $g_{z, \pi}$ and $g_{-z, \sigma(\pi)}$ commute. Let

$$
h_{z, \pi}=g_{z, \pi} g_{-z, \sigma(\pi)} .
$$

Note

$$
\sigma\left(\pi^{\perp}\right)=\sigma(\mathrm{I}-\pi)=\mathrm{I}-\sigma(\pi)=(\sigma(\pi))^{\perp} .
$$

Use (8.5), $\sigma\left(\pi^{\perp}\right)=\sigma(\pi)^{\perp}$ and a direction computation to prove that $h_{z, \pi}$ satisfies the $\frac{S U(n)}{S(U(n-1) \times U(1))}$-reality condition:

$$
h(\bar{\lambda})^{*} h(\lambda)=\mathrm{I}, \quad h(-\lambda)=\sigma(h(\lambda)) .
$$

We apply Bäcklund transformations given by these elements to the stationary wave maps (closed geodesics) to construct homoclinic wave maps from $S^{1} \times \mathbb{R}$ to $\mathbb{C} P^{n-1}$.

Let $a_{0} \in \mathcal{P}$ so that $e^{2 \pi a_{0}}=\mathrm{I}, m$ an integer, and $a=m a_{0}$. Since $a_{0} \in s u(n)$, there exists $A \in S U(n)$ and $C=\operatorname{diag}\left(i c_{1}, \ldots, i c_{n}\right)$ so that $a_{0}=A C A^{-1}$. Because $e^{2 \pi a_{0}}=\mathrm{I}$, all $c_{j}$ must be integers. Note $(a, 0, a)$ is a solution of the -1 flow equation associated to $S U(n)$ with $a \in \mathcal{P}$, and the corresponding wave map constructed in Theorem 4.2 is

$$
f_{0}(x, t)=e^{-2 a x}=A e^{-2 C x} A^{-1} \in M_{\sigma}=\mathbb{C} P^{n-1} .
$$

Choose $z_{1}, \ldots, z_{j} \in \mathbb{C}$ so that

$$
z_{j}=e^{i \theta_{j}}=\frac{r_{j}+i \mu_{j}}{m}
$$


as in Example 5.4. Let

$$
\begin{aligned}
h_{z, \pi} \bullet(a, u, v) & =g_{z, \pi} \bullet\left(g_{-z, \sigma(\pi)} \bullet(a, u, v)\right), \\
\left(a, u_{j}, v_{j}\right) & =h_{z_{j}, \pi} \bullet\left(\cdots \bullet\left(h_{z_{1}, \pi} \bullet(a, 0, a)\right) \cdots\right), \\
f_{j} & =h_{z_{j}, \pi} \bullet\left(\cdots \bullet\left(h_{z_{1}, \pi} \bullet f_{0}\right) \cdots\right) .
\end{aligned}
$$

The computation in Example 5.4 and the proof of Theorem 6.1 implies that $f_{k}$ is a homoclinic $2 k$-soliton wave map from $S^{1} \times \mathbb{R}^{1}$ to $S U(n)$. But each $h_{z_{j}, \pi_{j}}$ satisfies the reality conditions (8.7). Use a proof similar to that of Proposition 7.3(2) to see that $v_{j} \in \mathcal{P}$ and $u_{j} \in \mathcal{K}$ and the wave map corresponding to $\left(a, u_{j}, v_{j}\right)$ lies in $M_{\sigma}=\mathbb{C} P^{n-1}$. So $f_{j}$ is a wave map from $S^{1} \times \mathbb{R}$ to $S U(n)$ whose image lies in $\mathbb{C} P^{n-1}$. By Theorem 6.1, $f_{k}$ is a homoclinic wave map from $S^{1} \times \mathbb{R}$ to $\mathbb{C} P^{n-1}$.

Example 8.3. Wave maps from $S^{1} \times \mathbb{R}^{1}$ to $\mathbb{R} P^{n-1}$ and $S^{n-1}$

Let $G=S O(n, \mathbb{C})$,

$$
\tau(g)=\bar{g}, \quad \sigma(g)=J g J^{-1},
$$

where $J=\operatorname{diag}(1, \ldots, 1,-1)$. It can be checked easily that both $\tau$ and $\sigma$ are group homomorphisms of $G, \tau^{2}=\sigma^{2}=\mathrm{Id}$ and $\tau \sigma=\sigma \tau$. The fixed point set of $\tau$ in $G$ is $U=S O(n)$, and the fixed point set of $\sigma$ in $U$ is $S(O(1) \times O(n-1))$. The \pm 1 eigenspace of $\sigma_{*}$ in $\mathcal{U}$ is

$$
\begin{aligned}
& \mathcal{K}=\left\{\left(\begin{array}{ll}
0 & 0 \\
0 & \xi
\end{array}\right) \mid \xi \in s o(n-1)\right\} \\
& \mathcal{P}=\left\{\left(\begin{array}{cc}
0 & v^{t} \\
-v & 0
\end{array}\right) \mid v \in \mathcal{M}_{1 \times(n-1)}(\mathbb{R})\right\} .
\end{aligned}
$$

The symmetric space corresponding to $\tau, \sigma$ is $\frac{S O(n)}{S(O(n) \times O(1))}$, which is $\mathbb{R} P^{n-1}$. Let $g=\left(\begin{array}{ll}D & b \\ v & c\end{array}\right) \in S O(n)$ with $D \in g l(n-1, \mathbb{R}), b \in \mathcal{M}_{(n-1) \times 1} *(\mathbb{R})$, $v \in \mathcal{M}_{1 \times(n-1)}(\mathbb{R})$, and $c \in \mathbb{R}$. A direct computation gives

$$
g \sigma(g)^{-1}=\left(\begin{array}{cc}
\mathrm{I}-2 b b^{t} & 2 b c \\
-2 c b^{t} & 2 c^{2}-1
\end{array}\right) .
$$

So the Cartan embedding is

$$
\begin{aligned}
M_{\sigma} & =\left\{g \sigma(g)^{-1}=g J g J^{-1} \mid g \in S O(n)\right\} \\
& =\left\{\left(\begin{array}{cc}
\mathrm{I}-2 b b^{t} & 2 b c \\
-2 c b^{t} & 2 c^{2}-1
\end{array}\right) \mid b \in \mathcal{M}_{(n-1) \times 1}(\mathbb{R}), c \in \mathbb{R}, c^{2}+\|b\|^{2}=1\right\} .
\end{aligned}
$$


The map from

$$
S^{n-1}=\left\{\left.\left(\begin{array}{l}
b \\
c
\end{array}\right)\left|c \in \mathbb{R}, b \in \mathbb{R}^{n-1},\right| c\right|^{2}+\|b\|^{2}=1\right\}
$$

to $M_{\sigma}$ defined by

$$
\left(\begin{array}{l}
b \\
c
\end{array}\right) \mapsto\left(\begin{array}{cc}
\mathrm{I}-2 b b^{t} & 2 b c \\
-2 c b^{t} & 2 c^{2}-1
\end{array}\right)
$$

is a double covering. This shows that $M_{\sigma}$ is isometric to $\mathbb{R} P^{n-1}$.

To construct explicit wave maps into $S^{n-1}$ from a stationary wave map into $S^{n-1}$, we need to find rational maps $g$ from $S^{2}$ to $S O(n, \mathbb{C})$ that satisfying $g(\infty)=\mathrm{I}$ and the $\frac{S O(n)}{S(O(n-1) \times O(1))}$-reality conditions:

$$
\overline{g(\bar{\lambda})} g(\lambda)=\mathrm{I}, \quad g(-\lambda)=J g(\lambda) J^{-1} .
$$

This is equivalent to find rational maps from $S^{2}$ to $G L(n, \mathbb{C})$ that satisfies

$$
g(\bar{\lambda})^{*} g(\lambda)=\mathrm{I}, \quad \overline{g(\bar{\lambda})} g(\lambda)=\mathrm{I}, \quad g(-\lambda)=J g(\lambda) J^{-1} .
$$

We have seen in Example 8.2 that $h_{z, \pi}$ defined by (8.6) satisfies the first and third conditions of (8.9), but in general it does not satisfies the second condition. However, let $w \in \mathbb{R}^{n-1}$ and $b \in \mathbb{R}$ so that $\|w\|=|b|=1$, and $\pi$ the projection onto $\mathbb{C}\left(\begin{array}{l}w \\ i b\end{array}\right)$. A direct computation shows that

$$
\pi=\left(\begin{array}{cc}
w w^{t} & -i b w \\
i b w^{t} & 1
\end{array}\right), \quad \sigma(\pi)=\bar{\pi}
$$

By (8.5), we have

$$
\pi \bar{\pi}=\bar{\pi} \pi=0 .
$$

It is easy to check that

$$
\phi_{z, \pi}=h_{z, \pi} h_{-\bar{z}, \pi}=g_{z, \pi} g_{-z, \bar{\pi}} g_{-\bar{z}, \pi} g_{\bar{z}, \bar{\pi}}
$$

satisfies all conditions in (8.9). Note $\phi$ has four simple poles, $z,-z, \bar{z},-\bar{z}$.

Let $a_{0} \in \mathcal{P}$ so that $e^{2 \pi a_{0}}=\mathrm{I}$. Then $(a, 0, a)$ is a solution of the -1 flow equation associated to $S O(n)$ whose corresponding wave map is the stationary wave map into $M_{\sigma}: s_{0}(x, t)=e^{-2 a_{0} x}$. Let $m$ an integer, and

$$
a=m a_{0}
$$


Let

$$
z_{j}=e^{i \theta_{j}}=\frac{r_{j}+i \mu_{j}}{m}
$$

where $r_{j}$ is an integer. Let

$$
\phi \bullet(a, u, v)=h_{z, \pi} \bullet\left(h_{-\bar{z}, \pi} \bullet(a, u, v)\right) .
$$

Use similar reasoning as in Example 8.2 to see that wave map

$$
s_{j}=\phi_{z_{j}, \pi} \bullet\left(\cdots\left(\phi_{z_{1}, \pi} \bullet s_{0}\right) \cdots\right)
$$

is a homoclinic $4 j$-soliton wave map from $S^{1} \times \mathbb{R}^{1}$ to $\mathbb{R} P^{n-1}$. Moreover, the last column of $s_{j}$ gives a homoclinic wave map from $S^{1} \times \mathbb{R}^{1}$ to $S^{n-1}$.

\section{Wave maps from $\mathbb{R}^{1,1}$ to $S L(2, \mathbb{R})$.}

It is known that the Cauchy problem for wave map equation from $\mathbb{R}^{1,1}$ to any complete Riemannian manifold has long time existence ([1]). We will show that this is no longer true when the target manifold is the pseudoRiemannian manifold $S L(2, \mathbb{R})$. In fact, we use Bäcklund transformations to construct smooth initial data with finite energy and constant boundary condition at $\pm \infty$ so that the Cauchy problem for wave maps into $S L(2, \mathbb{R})$

1. has long time existence, or

2. develops singularities in finite time.

First note that Theorem 4.2 holds if we replace $S U(n)$ by any group $G$. But Bäcklund transformations for the -1 flow equation associated to $S L(2, \mathbb{R})$ is different from the $S U(n)$ case. Let $\alpha_{1}, \alpha_{2} \in \mathbb{C}$, and $\pi$ a linear projection of $\mathbb{C}^{n}$ (i.e., $\pi$ is complex linear and $\pi^{2}=\pi$ ). Let

$$
h_{\alpha_{1}, \alpha_{2}, \pi}(\lambda)=\mathrm{I}+\frac{\alpha_{1}-\alpha_{2}}{\lambda-\alpha_{1}} \pi^{\prime},
$$

where $\pi^{\prime}=\mathrm{I}-\pi$. Then

$$
h_{\alpha_{1}, \alpha_{2}, \pi}^{-1}(\lambda)=\mathrm{I}+\frac{\alpha_{2}-\alpha_{1}}{\lambda-\alpha_{2}} \pi^{\prime} .
$$

Bäcklund transformations are given as follows (cf. [5]): 
Theorem 9.1. Let $(a, u, v)$ be a smooth solution of the -1 flow equation associated to $S L(n, \mathbb{C}), \theta_{\lambda}=(a \lambda+u) d \xi+v \lambda^{-1} d \eta$ its Lax pair, and $E$ the trivialization of $\theta_{\lambda}$. Let $\pi$ be the projection of $\mathbb{C}^{n}, V_{1}=\operatorname{Im}(\pi)$, and $V_{2}=\operatorname{Ker}(\pi)$. Set

$$
\tilde{V}_{i}(\xi, \eta)=E\left(\xi, \eta, \alpha_{i}\right)^{-1}\left(V_{i}\right), \quad \text { for } i=1,2 .
$$

Suppose $\tilde{V}_{1}(\xi, \eta) \cap \tilde{V}_{2}(\xi, \eta)=\{0\}$ for $(\xi, \eta)$ lies in an open subset $\mathcal{O}$ of $\mathbb{R}^{2}$. Let $\tilde{\pi}(\xi, \eta)$ denote the linear projection onto $\tilde{V}_{1}(\xi, \eta)$ along $\tilde{V}_{2}(\xi, \eta)$, and

$$
\begin{aligned}
\tilde{u} & =u+\left(\alpha_{1}-\alpha_{2}\right)[a, \tilde{\pi}], \\
\tilde{v} & =\left(\mathrm{I}-\frac{\alpha_{1}-\alpha_{2}}{\alpha_{1}} \tilde{\pi}\right) v\left(\mathrm{I}-\frac{\alpha_{1}-\alpha_{2}}{\alpha_{1}} \tilde{\pi}\right)^{-1} \\
& =\left(-\alpha_{1} \tilde{\pi}+\alpha_{2} \tilde{\pi}^{\prime}\right) v\left(-\alpha_{1}^{-1} \tilde{\pi}+\alpha_{2}^{-1} \tilde{\pi}^{\prime}\right) .
\end{aligned}
$$

Then $(a, \tilde{u}, \tilde{v})$ is a smooth solution of the -1 flow equation associated to $S L(n, \mathbb{C})$ defined on $\mathcal{O}$. Moreover, the trivialization of the Lax pair of $(a, \tilde{u}, \tilde{v})$ is

$$
\tilde{E}(\xi, \eta, \lambda)=h_{\alpha_{1}, \alpha_{2}, \pi}(\lambda) E(\xi, \eta, \lambda) h_{\alpha_{1}, \alpha_{2}, \tilde{\pi}(x, t)}(\lambda)^{-1} .
$$

We use $h_{\alpha_{1}, \alpha_{2}, \pi} \bullet(a, u, v)$ to denote $(a, \tilde{u}, \tilde{v})$.

If $(a, u, v)$ is a solution of the -1 flow equation associated to $S L(n, \mathbb{R})$, then $h_{\alpha_{1}, \alpha_{2}, \pi} \bullet(a, u, v)$ is also a solution of the -1 flow equation associated to $S L(n, \mathbb{R})$ provided that $\alpha_{1}, \alpha_{2} \in \mathbb{R}$ and $\bar{\pi}=\pi$.

Example 9.1. Let $a=\operatorname{diag}(1,-1)$. Then $(a, 0, a)$ is a solution of the -1 flow equation associated to $S L(2, \mathbb{R})$ and the corresponding wave map is $s(x, t)=e^{-2 a x}$. Let $\alpha_{1}, \alpha_{2} \in \mathbb{R}, v_{1}=\left(\begin{array}{l}c_{1} \\ c_{2}\end{array}\right) \in \mathbb{R}^{2}, v_{2}=\left(\begin{array}{l}d_{1} \\ d_{2}\end{array}\right) \in \mathbb{R}^{2}$, and $\pi$ the projection of $\mathbb{C}^{2}$ onto $\mathbb{C} v_{1}$ along $\mathbb{C} v_{2}$. Let

$$
(a, \tilde{u}, \tilde{v})=h_{\alpha_{1}, \alpha_{2}, \pi} \bullet(a, 0, a) .
$$

Use Theorem 9.1 and a direct computation to get

$$
\tilde{\pi}=\frac{1}{c_{1} d_{2} e^{A}-c_{2} d_{1} e^{-A}}\left(\begin{array}{ll}
c_{1} d_{2} e^{A} & -c_{1} d_{1} e^{-B} \\
c_{2} d_{2} e^{B} & -c_{2} d_{1} e^{-A}
\end{array}\right),
$$

where

$$
A=\left(\alpha_{2}-\alpha_{1}\right) \xi+\left(\alpha_{2}^{-1}-\alpha_{1}^{-1}\right) \eta, \quad B=\left(\alpha_{2}+\alpha_{1}\right) \xi+\left(\alpha_{2}^{-1}+\alpha_{1}^{-1}\right) \eta .
$$


The new solution $(a, \tilde{u}, \tilde{v})$ is expressed in terms of $\tilde{\pi}(\xi, \eta)$ as given in Theorem 9.1. The wave map corresponding to $(a, \tilde{u}, \tilde{v})$ is

$$
\begin{aligned}
\tilde{s}(\xi, \eta) & =\tilde{E}(\xi, \eta,-1) \tilde{E}(\xi, \eta, 1)^{-1} \\
& =h(-1) e^{-a x}\left(\frac{\left(1+\alpha_{1}\right)\left(1-\alpha_{2}\right)-2\left(\alpha_{1}-\alpha_{2}\right) \tilde{\pi}(x, t)}{\left(1+\alpha_{2}\right)\left(1-\alpha_{1}\right)}\right) e^{-a x} h(1)^{-1},
\end{aligned}
$$

where $h(\lambda)=h_{\alpha_{1}, \alpha_{2}, \pi}(\lambda)$. If $\tilde{\pi}$ has singularities, then $(a, \tilde{u}, \tilde{v})$ and $\tilde{s}$ have too. Note that we can choose $\alpha_{i}$ and $v_{1}, v_{2}$ so that $h_{\alpha_{1}, \alpha_{2}, \pi} \bullet(a, 0, a)$ is singular somewhere or is smooth on the whole $\mathbb{R}^{1,1}$. But such wave maps do not have good boundary behavior when $x \rightarrow \pm \infty$.

Example 9.2. We construct wave maps whose image lies in the subgroup

$$
\mathbb{R}^{+}=\left\{\operatorname{diag}\left(e^{t}, e^{-t}\right) \mid t \in \mathbb{R}\right\}
$$

of $S L(2, \mathbb{R})$. Let $h(\xi)$ and $k(\eta)$ be smooth real valued functions, and

$$
a(\xi)=h^{\prime}(\xi) \operatorname{diag}(1,-1), \quad b(\eta)=k^{\prime}(\eta) \operatorname{diag}(1,-1) .
$$

Then $(a, 0, b)$ is a solution of the -1 flow equation associated to $S L(2, \mathbb{R})$, its Lax pair is $\theta_{\lambda}=a(\xi) \lambda d \xi+b(\eta) \lambda^{-1} d \eta$, and the trivialization of $\theta_{\lambda}$ is

$$
E(\xi, \eta, \lambda)=\operatorname{diag}\left(e^{h(\xi) \lambda+k(\eta) \lambda^{-1}}, e^{-\left(h(\xi) \lambda+k(\eta) \lambda^{-1}\right)}\right) .
$$

The corresponding wave map is

$$
s(\xi, \eta)=E(\xi, \eta,-1) E(\xi, \eta, 1)^{-1}=\operatorname{diag}\left(e^{-2(h(\xi)+k(\eta))}, e^{2(h(\xi)+k(\eta))}\right) .
$$

Since the subgroup $\mathbb{R}^{+}$is abelian, the equation for wave maps into $\mathbb{R}^{+}$ is the linear wave equation. Hence every wave maps into $\mathbb{R}^{+}$is of the form given in (9.2) for some smooth one variable real valued functions $h, k$.

Example 9.3. We compute Bäcklund transformation of the wave maps given in Example 9.2. Let $\alpha_{1}, \alpha_{2} \in \mathbb{R}$, and $\pi$ the projection of $\mathbb{C}^{2}$ onto $\mathbb{C} y_{1}$ along $\mathbb{C} y_{2}$, where $y_{1}=\left(\begin{array}{l}c_{1} \\ d_{1}\end{array}\right)$ and $y_{2}=\left(\begin{array}{l}c_{2} \\ d_{2}\end{array}\right)$ are in $\mathbb{R}^{2}$. To get $h_{\alpha_{1}, \alpha_{2}, \pi} \bullet(a, 0, b)$, we first compute

$$
\begin{aligned}
& \tilde{y}_{1}(\xi, \eta)=E\left(\xi, \eta, \alpha_{1}\right)^{-1}\left(y_{1}\right)=\left(\begin{array}{c}
c_{1} e^{-A_{1}} \\
d_{1} e^{A_{1}}
\end{array}\right), \\
& \tilde{y}_{2}(\xi, \eta)=E\left(\xi, \eta, \alpha_{2}\right)^{-1}\left(y_{2}\right)=\left(\begin{array}{c}
c_{2} e^{-A_{2}} \\
d_{2} e^{A_{2}}
\end{array}\right),
\end{aligned}
$$


where

$$
A_{i}=h(\xi) \alpha_{i}+k(\eta) \alpha_{i}^{-1} \quad \text { for } i=1,2 .
$$

Let $\tilde{\pi}(\xi, \eta)$ be the projection onto $\mathbb{C} \tilde{y}_{1}(\xi, \eta)$ along $\mathbb{C} \tilde{y}_{2}(\xi, \eta)$. Then

$$
\tilde{\pi}=\frac{1}{W}\left(\begin{array}{cc}
c_{1} d_{2} e^{-A_{1}+A_{2}} & -c_{1} c_{2} e^{-\left(A_{1}+A_{2}\right)} \\
d_{1} d_{2} e^{A_{1}+A_{2}} & -c_{2} d_{1} e^{A_{1}-A_{2}}
\end{array}\right),
$$

where

$$
W:=c_{1} d_{2} e^{-A_{1}+A_{2}}-c_{2} d_{1} e^{A_{1}-A_{2}} .
$$

So $(a, \tilde{u}, \tilde{v}):=h_{\alpha_{1}, \alpha_{2}, \pi} \bullet(a, 0, b)$ is given by

$$
\left\{\begin{array}{l}
\tilde{u}=\left(\alpha_{1}-\alpha_{2}\right)[a, \tilde{\pi}] \\
\tilde{v}=\left(\mathrm{I}-\frac{\alpha_{1}-\alpha_{2}}{\alpha_{1}} \tilde{\pi}\right) b\left(\mathrm{I}-\frac{\alpha_{1}-\alpha_{2}}{\alpha_{1}} \tilde{\pi}\right)^{-1}
\end{array}\right.
$$

The trivialization of $(a, \tilde{u}, \tilde{v})$ is

$$
\tilde{E}(\xi, \eta, \lambda)=h_{\alpha_{1}, \alpha_{2}, \pi}(\lambda) E(\xi, \eta, \lambda) h_{\alpha_{1}, \alpha_{2}, \tilde{\pi}(\xi, \eta)}^{-1}(\lambda),
$$

and the corresponding wave map is

$$
\tilde{s}(\xi, \eta)=h(-1) A_{0}\left(\frac{\left(1+\alpha_{1}\right)\left(1-\alpha_{2}\right)-2\left(\alpha_{1}-\alpha_{2}\right) \tilde{\pi}}{\left(1+\alpha_{2}\right)\left(1-\alpha_{1}\right)}\right) A_{0} h(1)^{-1},
$$

where $h(\lambda)=h_{\alpha_{1}, \alpha_{2}, \pi}(\lambda)$ and $A_{0}(\xi, \eta)=\operatorname{diag}\left(e^{-(h(\xi)+k(\eta))}, e^{(h(\xi)+k(\eta))}\right)$.

It can be easily checked that

(i) $\tilde{\pi}(\xi, \eta)$ has singularity at $\left(\xi_{0}, \eta_{0}\right)$ if and only if $W\left(\xi_{0}, \eta_{0}\right)=0$. For example, if $\frac{c_{1} d_{2}}{c_{2} d_{1}}>0$, then $\tilde{\pi}$ has singularities at points on the curve

$$
h(\xi)\left(\alpha_{1}-\alpha_{2}\right)+k(\eta)\left(\alpha_{1}^{-1}-\alpha_{2}^{-1}\right)-\frac{1}{2} \ln \left(\frac{c_{1} d_{2}}{c_{2} d_{1}}\right)=0 .
$$

(ii) If $\frac{c_{1} d_{2}}{c_{2} d_{1}}<0$, then $W$ never vanishes. So $\tilde{\pi}$ is smooth for all $(\xi, \eta)$.

Now suppose that both $h$ and $k$ is in $L_{1}^{2}$, i.e, $h, k, h^{\prime}, k^{\prime}$ are square integrable. If $W\left(x, t_{0}\right) \neq 0$ for all $x \in \mathbb{R}$, then the formula for $\tilde{\pi}$ implies that

$$
\lim _{|x| \rightarrow \infty} \tilde{\pi}\left(x, t_{0}\right)=\frac{1}{c_{1} d_{2}-c_{2} d_{1}}\left(\begin{array}{cc}
c_{1} d_{2} & -c_{1} c_{2} \\
d_{1} d_{2} & -d_{1} c_{2}
\end{array}\right)=\pi
$$

So

$$
\lim _{|x| \rightarrow-\infty} \tilde{s}\left(x, t_{0}\right)=h(-1)\left(\frac{\left(1+\alpha_{1}\right)\left(1-\alpha_{2}\right)-2\left(\alpha_{1}-\alpha_{2}\right) \pi}{\left(1+\alpha_{2}\right)\left(1-\alpha_{1}\right)}\right) h(1)^{-1}
$$


is a constant

Rewrite the left hand side of (9.4) in space time coordinates $x, t$, and set

$$
f(x, t)=\left(\alpha_{1}-\alpha_{2}\right) h((x+t) / 2)-\left(\alpha_{1}^{-1}-\alpha_{2}^{-1}\right) k((x-t) / 2)-\frac{1}{2} \ln \left(\frac{c_{1} d_{2}}{c_{2} d_{1}}\right)
$$

For case (i), we can choose $\alpha_{1}, \alpha_{2} \in \mathbb{R}$ and $h, k$ in $L_{1}^{2}$ so that $f(x, 0)$ never vanishes for all $x \in \mathbb{R}$, but vanishes at some $\left(x_{0}, t_{0}\right)$ for some $t_{0}>0$. We check that the wave map $\tilde{s}(x, 0)$ is smooth with finite energy and constant boundary condition, but it develops singularities in finite time.

For case (ii), we have proved that $\tilde{s}(x, t)$ has constant boundary condition for all $t$. Claim that the energy of $\tilde{s}$ is finite. To see this, note that by Theorem 4.2 we have

$$
\begin{aligned}
& \operatorname{tr}\left(\tilde{s}^{-1} \tilde{s}_{\xi}, \tilde{s}^{-1} s_{\xi}\right)=4 \operatorname{tr}(a, a), \\
& \operatorname{tr}\left(\tilde{s}^{-1} \tilde{s}_{\eta}, \tilde{s}^{-1} \tilde{s}_{\eta}\right)=4 \operatorname{tr}(\tilde{v}, \tilde{v})
\end{aligned}
$$

$\operatorname{But} \operatorname{tr}(\tilde{v}, \tilde{v})=\operatorname{tr}(b, b)$, which is finite. Thus $\tilde{s}$ is smooth, $\lim _{|x| \rightarrow \infty} \tilde{s}(x, t)$ is a fixed constant for all $t$, and has finite energy.

\section{References.}

[1] Gu, C. H., On the Cauchy problem for harmonic maps defined on twodimensional Minkowski space, Comm. Pure Appl. Math. 33 (1980), 727737

[2] Pohlmeyer, K., Integrable Hamiltonian systems and interactions through quadratic constraints, Comm. Pure Appl. Math., 46 (1976), 207-221

[3] Shatah, J., Strauss, W., Breathers as homoclinic geometric wave maps, Physics, D, 99 (1996), 113-133

[4] Terng, C.L., Uhlenbeck, K., Schrödinger flows on Grassmannians, to appear in "Integrable systems, Geometry, and Topology", International Press, math.DG/9901086

[5] Terng, C.L., Uhlenbeck, K., Bäcklund transformations and loop group actions, Comm. Pure. Appl. Math., 53 (2000), 1-75

[6] Uhlenbeck, K., Harmonic maps into Lie group (classical solutions of the Chiral model), J. Differential Geometry, 30 (1989), 1-50 
[7] Zakharov, V.E., Mikhailov, A.V., Relativistically invariant twodimensional models of field theory which are integrable by means of the inverse scattering problem method, Sov. Phys. JETP, 47 (1978), 10171027

Chuu-Lian Terng

Department of Mathematics

NORTHEASTERN UNIVERSITY

Boston, MA 02115

terng@neu. eud

KAREN UhLENBECK

Department of Mathematics

University of Texas at Austin

Austin, TX 78712

uhlen@math . utexas .edu 